Mon. Not. R. Astron. Soc. 000, 000-000 (1994) Printed 21 March $2018 \quad$ (MN plain TEX macros v1.6)

\title{
The Effect of Encounters on the Eccentricity of Binaries in Clusters
}

\author{
Douglas C. Heggie ${ }^{1}$ and Frederic A. Rasio ${ }^{2}$ \\ ${ }^{1}$ Department of Mathematics and Statistics, University of Edinburgh, King's Buildings, Edinburgh EH9 3JZ, UK. Email: \\ d.c.heggie@ed.ac.uk \\ 2 Department of Physics, M.I.T., Cambridge, Massachusetts 02139, USA. Email: rasio@mit.edu
}

\begin{abstract}
We derive analytical expressions for the change in the orbital eccentricity of a binary following a distant encounter with a third star on a hyperbolic or parabolic orbit. To establish the accuracy of these expressions, we present detailed comparisons with the results of direct numerical integrations of the equations of motion for the three bodies. We treat with particular care the difficult case of a binary with zero initial eccentricity. In this case, we show that the eccentricity $\delta e$ induced by the encounter declines in general as a power-law, $\delta e \propto\left(a / r_{\mathrm{p}}\right)^{5 / 2}$, where $a$ is the binary semi-major axis and $r_{\mathrm{p}}$ is the periastron distance of the encounter. This power-law arises from the octupole-level secular perturbation of the binary. In contrast, non-secular quadrupolelevel perturbations induce an eccentricity change that declines exponentially with $r_{\mathrm{p}}$. These non-secular effects can become dominant at sufficiently small $r_{\mathrm{p}}$, for a sufficiently high relative velocity, or for a sufficiently massive perturber. We also derive cross sections for eccentricity change and compare our results with those of previous studies based on numerical scattering experiments. Our results have important implications for a number of astrophysical problems including, in particular, the evolution of binary millisecond pulsars in globular clusters.
\end{abstract}

Key words: binaries: close - celestial mechanics, stellar dynamics - globular clusters: general - pulsars: general

\section{INTRODUCTION}

Three-body scattering plays an essential role in stellar dynamics. In particular the interactions between binary stars and single stars in globular clusters are thought to be an important dynamical process, providing support against core collapse (Spitzer 1987; Hut et al. 1992). What is important here is the energy exchanged, but there are applications in which it is also important to understand the changes in the eccentricity of a binary caused by an encounter with another star. In some cases even very small changes in eccentricity can be very significant. Most importantly, binary millisecond pulsars have been discovered in large numbers in globular clusters and their orbital eccentricities can be measured with extraordinary precision. Although the orbits of binary millisecond pulsars in the galactic plane are nearly circular (for example, PSR J2317+1439 has a measured eccentricity of $1.2 \times 10^{-6}$; see Camilo et al. 1993), those found in the dense stellar environment of globular clusters may be perturbed by the high rate of encounters with other stars. If, therefore, the rate at which the eccentricity is changed by encounters is known, interesting constraints can sometimes be placed on the lifetimes of the binaries (Rappaport et al. 1989; Rasio \& Heggie 1995).

Much of our knowledge of the effect of encounters on eccentricity is numerical (Hills 1975; Hills \& Fullerton 1980; Hut \& Paczyński 1984; Rappaport et al. 1989). In the present paper the approach is analytical, with numerical work used only to confirm the theoretical results. Heggie (1975) gave a theoretical estimate (based on first-order perturbation theory) for the change in eccentricity, and found that it varies inversely as the $3 / 2$ power of $r_{\mathrm{p}}$, the distance of closest approach of the third body. Unfortunately, his result vanishes in the important case in which the initial orbit is circular. Phinney (1992) and Heggie (unpublished) then found, again analytically, that in this case the induced eccentricity appeared to decrease exponentially with a power of $r_{\mathrm{p}}$, though the results were restricted to special situations (e.g., equal masses, coplanar motions, and parabolic or rectilinear motion of the third body). While the authors of the present paper were reworking the theory for more general cases, S.L.W. McMillan pointed out to us, on the basis of numerical experiments with a new scattering package (McMillan 1996), that the predicted exponential dependence for initially circular binaries fails when the masses of the binary components are unequal (which clearly is the important case in applications). This discovery stimulated much of the remaining work for 
the present paper.

In this paper our aim is to clarify the regimes in which the various theoretical results are applicable, and to extend them, where necessary, to arbitrary masses and arbitrary geometries. The one common assumption is that the encounter is tidal, i.e., that $r_{\mathrm{p}} \gg a$, where $a$ is the initial semi-major axis of the binary, though it may be necessary to take account of the masses of the participants in this condition when the masses are very different. We also usually assume that the encounter is slow.

In the following section we outline the theoretical results, but leave detailed derivations to an appendix. We also compare the theoretical results with new numerical data, designed to explore the full dependence on $r_{\mathrm{p}}$, the masses, the initial eccentricity of the binary, and the geometry of the orbits. In Section 3 we convert our results into cross sections, which are then compared with results of other authors. In the last section we summarise our conclusions and outline briefly some applications. Another summary, which places particular emphasis on applications to binary pulsars in globular clusters, can be found in Rasio \& Heggie (1995).

\section{GENERAL EXPRESSIONS FOR THE CHANGE IN ECCENTRICITY}

\subsection{Notations, Assumptions and Basic Equations}

After setting out our notation and assumptions, in this section we shall give the basic equations for the perturbation of a binary by a third body. Much of this is elementary, but it is helpful because it makes some properties of our detailed results quite obvious.

We consider an encounter between a single star of mass $m_{3}$ and a binary whose components have masses $m_{1}$ and $m_{2}$, and we define $M_{12}=m_{1}+m_{2}$ and $M_{123}=M_{12}+m_{3}$. Long before the encounter begins, the eccentricity of the binary is $e$, (though often we shall assume this is zero), and its semi-major axis is $a$. The orbit of the third body relative to the barycentre of the binary is hyperbolic with eccentricity $e^{\prime}$. We denote its impact parameter by $p$ and its speed relative to the barycentre, while they are still far apart, by $V$. Then we have

$e^{\prime}=\sqrt{1+\frac{p^{2} V^{4}}{G^{2} M_{123}^{2}}}$

and we shall be able to obtain results for a parabolic encounter by letting $V \rightarrow 0$, or $e^{\prime} \rightarrow 1$.

We make two assumptions about the encounter, that it is both tidal and slow. For the first of these, we assume that the closest distance of approach of the third body, which will be denoted by $r_{\mathrm{p}}$, is still considerably larger than $a$. The ratio of these distances will play the role of a perturbation parameter. For the second assumption, we require that the angular velocity of the perturber, when it is closest to the binary, is considerably smaller than the angular velocity of the binary components. It is easily shown that this is equivalent to the condition

$\sqrt{\frac{M_{12}}{M_{123}}}\left(\frac{r_{\mathrm{p}}}{a}\right)^{3 / 2} \frac{1}{\sqrt{e^{\prime}+1}} \gg 1$,

and so the second assumption is implied by the first unless the masses are very disparate or $e^{\prime}$ is very large. (This last case, the "impulsive limit", is briefly considered in Section 3.2 and Appendix A4.)

Now we develop the equations of motion. Let $\mathbf{r}$ be the position of $m_{2}$ relative to $m_{1}$, and $\mathbf{R}$ the position of $m_{3}$ relative to the barycentre of the binary. Then the equation for the relative motion of the binary components is

$\ddot{\mathbf{r}}=\frac{-G M_{12} \mathbf{r}}{r^{3}}+\mathbf{F}$

with

$\mathbf{F}=\frac{G m_{3}}{R} \sum_{n=0}^{\infty} \frac{m_{1}^{n-1}-\left(-m_{2}\right)^{n-1}}{M_{12}^{n-1}} \nabla_{\mathbf{r}}\left[\left(\frac{r}{R}\right)^{n} P_{n}\left(\frac{\mathbf{r} \cdot \mathbf{R}}{r R}\right)\right]$,

where $P_{n}$ is the $n$th Legendre polynomial.

Except for a factor $-m_{1} m_{2} / M_{12}$, equation (4) is simply the gradient of the mutual potential of the components of the binary and the third body, expressed as a multipole expansion in powers of $r / R$. The monopole and dipole terms (i.e., those given by $n=0$ and 1) make no contribution to $\mathbf{F}$, and will play no further part in this paper.

Our aim is to compute the change in eccentricity of the binary over the entire duration of the encounter with the third body. To do this we have found it convenient to use the "eccentric axis" (Pollard 1976), which is a vector also associated with the names of Laplace, Hamilton, Lenz and Runge (Goldstein 1980). For unperturbed Keplerian motion it is a constant vector which points along the major axis of the elliptic orbit of the binary components, and its magnitude is $e$. Its definition is

$\mathbf{e}=\frac{1}{G M_{12}} \dot{\mathbf{r}} \times(\mathbf{r} \times \dot{\mathbf{r}})-\frac{\mathbf{r}}{r}$. 
Using equation (3) it is easy to show that, in the presence of a perturbing acceleration $\mathbf{F}$, its variation is determined by

$G M_{12} \dot{\mathbf{e}}=2(\mathbf{F} \cdot \dot{\mathbf{r}}) \mathbf{r}-(\mathbf{r} \cdot \dot{\mathbf{r}}) \mathbf{F}-(\mathbf{F} \cdot \mathbf{r}) \dot{\mathbf{r}}$

(cf. also Burdet 1967). These equations are the basis of the theory described in Appendix A, but some of the results we shall now summarise are more easily understood with reference to these formulae.

\subsection{Binaries with Non-Zero Initial Eccentricity}

We begin with what would seem to be the most straightforward and general case, that of a binary of arbitrary initial eccentricity $e$. Also, since we are assuming that $r_{\mathrm{p}} \gg a$, it is natural to neglect all but the lowest non-vanishing term in equation (4). From what has been said, this is the quadrupole term $n=2$ in the interaction between the binary and the third body.

From the assumption that the encounter is slow $(\S 2.1)$ it follows that, to lowest order, perturbations may be computed by first averaging over the fast relative motion of the components of the binary. The resulting estimate of the change in eccentricity is given (with a sign error) in the case of parabolic motion of the third body by Heggie (1975, eq. [5.66]), and it is rederived in the general case (and with the correct sign) in Appendix A to the present paper.

In order to state this result, we introduce standard angles describing the relative orientation of the orbits. The line of nodes is defined to be the line of intersection of the orbital planes of the third body and the binary. Then the ascending node is the part of this line at which the third body crosses the plane of the binary in the direction of the angular momentum vector of the binary. Let $\Omega$ be the longitude of the ascending node, measured in the plane of motion of the binary (in the sense of the motion of its components) from the direction of pericentre of the binary. Let $i$ be the inclination of the two orbital planes, and let $\omega$ be the longitude of pericentre of the third body, measured in its plane of motion from the ascending node, in the sense of its motion round the binary. Then the result is that the change in eccentricity is (cf. Appendix A1, eq. [A4])

$$
\begin{aligned}
\delta e= & -\frac{15}{4} \frac{m_{3}}{\sqrt{M_{12} M_{123}}}\left(\frac{a}{r_{\mathrm{p}}}\right)^{3 / 2} \frac{e \sqrt{1-e^{2}}}{\left(1+e^{\prime}\right)^{3 / 2}} \times \\
& \times\left\{\sin ^{2} i \sin 2 \Omega\left[\arccos \left(-1 / e^{\prime}\right)+\sqrt{e^{\prime^{2}}-1}\right]+\frac{1}{3}\left[\left(1+\cos ^{2} i\right) \cos 2 \omega \sin 2 \Omega+2 \cos i \sin 2 \omega \cos 2 \Omega\right] \frac{\left(e^{\prime^{2}}-1\right)^{3 / 2}}{e^{\prime^{2}}}\right\} .
\end{aligned}
$$

This result is admittedly quite inelegant, but the result for parabolic motion of the third body is neater; in that case

$\delta e=-\frac{15 \pi}{16}\left(\frac{2 m_{3}^{2} a^{3}}{M_{123} M_{12} r_{\mathrm{p}}^{3}}\right)^{1 / 2} e \sqrt{1-e^{2}} \sin 2 \Omega \sin ^{2} i$

The stated dependence on the ratio of the distances $a / r_{\mathrm{p}}$ is easily understood by examination of equations (4) and (6). The quadrupole term in equation (4) is proportional to $a / r_{\mathrm{p}}^{3}$, and so the terms in equation (6) are proportional to $a^{3} /\left(\operatorname{Tr}_{\mathrm{p}}^{3}\right)$, where $T$ is the period of the binary. By Kepler's Third Law the duration of the encounter is of order $T\left(r_{\mathrm{p}} / a\right)^{3 / 2}$, and so the integration of equation (6) with respect to time gives a result proportional to $\left(a / r_{\mathrm{p}}\right)^{3 / 2}$, as we have found.

The dependence on $\Omega$ in equation (8) is also not hard to understand, at least for equal masses. Consider the case $\omega=0$, $i=90^{\circ}$, for example, and imagine the orbit-averaged binary as a distribution of mass extended along its major axis. If $\Omega=0$ or $90^{\circ}$ then the orbit of the third body is situated on a plane of symmetry of the binary, and can exert no net torque. This already suggests a dependence on $\sin 2 \Omega$. Furthermore, if $\Omega=45^{\circ}$, the torque increases the angular momentum of the binary, decreasing its eccentricity, which is consistent with the sign of the right side of equation (8). On the other hand neither the dependence on $i$ nor the independence on $\omega$ can be understood from such considerations, as equation (7) shows that the situation for hyperbolic encounters is different.

\subsection{Initially Circular Binaries}

\subsubsection{Secular Octupole Perturbation: The Power-Law Regime}

Unfortunately this lowest-order result vanishes if the initial eccentricity is zero. This is evident from equation (7) and can also be understood from a rather general point of view, as discussed in Section 2.3.3 and Appendix B. For a non-trivial result in this case it is necessary to include the octupole contribution to the interaction, i.e., the term with $n=3$ in equation (4). Details of the calculation are given in Appendix A2, and the result for the eccentricity induced in an initially circular binary 
may be stated as follows:

$$
\begin{aligned}
\delta e=\frac{15}{8} \frac{m_{3} \mid m_{1}-}{M_{12}^{2}} m_{2} \mid & \left(\frac{M_{12}}{M_{123}}\right)^{1 / 2}\left(\frac{a}{r_{\mathrm{p}}}\right)^{5 / 2} \frac{1}{e^{\prime^{3}}\left(1+e^{\prime}\right)^{5 / 2}} \times \\
\times\left\{\cos ^{2} i \sin ^{2} \omega\right. & {\left[f_{1}\left(e^{\prime}\right)\left(1-\frac{15}{4} \sin ^{2} i\right)+\frac{2}{15}\left(e^{\prime 2}-1\right)^{5 / 2}\left(1-5 \sin ^{2} \omega \sin ^{2} i\right)\right]^{2}+} \\
& \left.+\cos ^{2} \omega\left[f_{1}\left(e^{\prime}\right)\left(1-\frac{5}{4} \sin ^{2} i\right)+\frac{2}{15}\left(e^{\prime 2}-1\right)^{5 / 2}\left(1-5 \sin ^{2} \omega \sin ^{2} i\right)\right]^{2}\right\}^{1 / 2},
\end{aligned}
$$

where

$f_{1}\left(e^{\prime}\right)=e^{\prime^{4}} \arccos \left(-1 / e^{\prime}\right)+\frac{\sqrt{{e^{\prime 2}}^{2}-1}}{15}\left(-2+9 e^{\prime^{2}}+8 e^{\prime^{4}}\right)$.

Again the result for parabolic motion of the third body is less inelegant. In fact for this case we have

$\delta e=\frac{15 \pi}{32} \frac{m_{3}\left|m_{1}-m_{2}\right|}{M_{12}^{2}}\left(\frac{M_{12}}{2 M_{123}}\right)^{1 / 2}\left(\frac{a}{r_{\mathrm{p}}}\right)^{5 / 2}\left[\cos ^{2} i \sin ^{2} \omega\left(1-\frac{15}{4} \sin ^{2} i\right)^{2}+\cos ^{2} \omega\left(1-\frac{5}{4} \sin ^{2} i\right)^{2}\right]^{1 / 2}$.

As before, it is easy to understand the dependence of these results on $a / r_{\mathrm{p}}$. The absence of $\Omega$ in these results should also come as no surprise, since the binary is assumed to be circular, and we have averaged over the position of its components. Another point of interest is the fact that the results are proportional to $\left|m_{1}-m_{2}\right|$, and the reason for this is easily seen by examination of the $n=3$ (octupole) term in equation (4).

In general, for a circular binary with unequal masses, the result (9) is the dominant contribution to the induced eccentricity at sufficiently large $r_{\mathrm{p}}$. We refer to this as the "power-law regime", since the change in eccentricity is still proportional to a power of $r_{\mathrm{p}} / a$, although it is a higher power than in the case of non-zero initial eccentricity. In contrast, at smaller $r_{\mathrm{p}}$, or in the special case of equal masses, we show in the next section that the induced eccentricity drops exponentially with $r_{\mathrm{p}}$.

\subsubsection{Non-Secular Quadrupole Perturbation: The Exponential Regime}

In Section 2.2 we showed that, if we average over the motion of the binary and take only the quadrupole interaction, then the eccentricity induced in an initially circular binary vanishes. At small $r_{\mathrm{p}}$, however, the assumption on which the method of averaging is based breaks down, and here we re-examine the problem without averaging. The result turns out to be important in applications. The derivation is deferred to Appendix A3, where it is shown that

$$
\begin{aligned}
\delta e=3 \sqrt{2 \pi} \frac{m_{3} M_{12}^{1 / 4}}{M_{123}^{5 / 4}}\left(\frac{r_{\mathrm{p}}}{a}\right)^{3 / 4} \frac{\left(e^{\prime}+1\right)^{3 / 4}}{e^{\prime^{2}}} & \exp \left[-\left(\frac{M_{12}}{M_{123}}\right)^{1 / 2}\left(\frac{r_{\mathrm{p}}}{a}\right)^{3 / 2} \frac{\sqrt{e^{\prime 2}-1}-\arccos \left(1 / e^{\prime}\right)}{\left(e^{\prime}-1\right)^{3 / 2}}\right] \times \\
& \times \cos ^{2} \frac{i}{2}\left[\cos ^{4} \frac{i}{2}+\frac{4}{9} \sin ^{4} \frac{i}{2}+\frac{4}{3} \cos ^{2} \frac{i}{2} \sin ^{2} \frac{i}{2} \cos (4 \omega+2 \Omega)\right]^{1 / 2}
\end{aligned}
$$

Here $\Omega$ is defined somewhat differently than in Section 2.2: it is measured in the plane of motion of the binary, from its position at the time of closest approach of the third body. Therefore this definition, and this formula, includes a dependence on the phase of the binary. Again this result simplifies a little for parabolic motion of the third body, to

$$
\begin{aligned}
\delta e=3 \sqrt{2 \pi} \frac{m_{3} M_{12}^{1 / 4}}{M_{123}^{5 / 4}}\left(\frac{2 r_{\mathrm{p}}}{a}\right)^{3 / 4} & \exp \left[-\frac{2}{3}\left(\frac{2 M_{12}}{M_{123}}\right)^{1 / 2}\left(\frac{r_{\mathrm{p}}}{a}\right)^{3 / 2}\right] \times \\
& \times \cos ^{2} \frac{i}{2}\left[\cos ^{4} \frac{i}{2}+\frac{4}{9} \sin ^{4} \frac{i}{2}+\frac{4}{3} \cos ^{2} \frac{i}{2} \sin ^{2} \frac{i}{2} \cos (4 \omega+2 \Omega)\right]^{1 / 2} .
\end{aligned}
$$

The approximations used in the derivation of these formulae again make use of the assumption that $r_{\mathrm{p}} \gg a$. Indeed for sufficiently large $r_{\mathrm{p}}$ these results are negligible in comparison with equations (7) and (8) if the binary is initially non-circular, or with equations (9) and (11) if $e=0$ initially. Nevertheless it turns out to be an important pair of results, which are dominant in a small but significant range of near-encounter distances where the exponential term is not so small as to be masked by the previous results.

There is one situation in which equations (12) and (13) appear to dominate at all $r_{\mathrm{p}} \gg a$ : the case of a circular binary with equal masses. This is not a rigorous result, but we do show in Appendix B that the induced eccentricity vanishes with increasing $r_{\mathrm{p}}$ more quickly than any power of $a / r_{\mathrm{p}}$. We already remarked that the octupole results, equations (9) and (11), are proportional to the mass difference $\left|m_{1}-m_{2}\right|$.

Looking at equations (12) and (13) one is tempted to conclude that the eccentricity is a kind of adiabatic invariant. It is usual, however, to apply this term to a dynamical quantity whose variation is small when the system is subject to a large but slow perturbation. Here the perturbation is not only slow but small. Furthermore, the exponential form of equations (12) 
and (13) only applies to a tiny set of orbits in which the initial eccentricity vanishes, whereas adiabatic invariants enjoy their special properties more globally. Henceforth we refer to this result simply as "the exponential formula", and when it is dominant we say that we are in the "exponential regime".

It is not as easy as in the previous cases to understand the dependence of these results on $a / r_{\mathrm{p}}$, except for the exponential term. The exponent is simply of order the ratio of the two main timescales in the problem: the period of the binary and the duration of the encounter. Such a term arises in other well studied problems of this kind (cf. Goldstein 1980, eq. [11-138]).

Finally, although the results in equations (12) and (13) vanish in the retrograde case $\left(i=180^{\circ}\right)$, this simply means that the leading term in this case is of a higher order in $a / r_{\mathrm{p}}$, and we have neglected all such terms in the derivation of these results.

\subsubsection{Remarks on First-Order versus Second-Order Perturbations}

Details of the derivation of the foregoing results are given in Appendix A, but it is worth exposing here one or two analytical issues which help to clarify the nature of the results.

There are several methods by which the computation of the change in eccentricity might be attempted. As already stated in Section 2.1, we have used the eccentric axis (Pollard 1976). Other possible methods are the use of Lagrange's planetary equations, though in their usual form they are unsuited for computations of low-eccentricity orbits, and it is better to express them in terms of Poincaré variables (Plummer 1918). From a physical point of view the most obvious method is to compute the change in angular momentum. This involves the changes in both $e$ and $a$, but the latter is already known (Heggie 1975, eq. [5.40]), and so the change in $e$ alone may be determined.

Whichever approach is adopted, the next step is a method of successive approximations. At lowest (first) order, unperturbed Keplerian motions are substituted into the perturbation terms. At next order, the first-order results are substituted, and so on. In addition, it is possible to express the perturbation term itself as a multipole expansion.

As we have seen, if we work to lowest order, retain only the quadrupole interaction, and average over the fast motion of the binary, then the eccentricity induced in a circular binary vanishes. It is tempting to offer the following explanation. After averaging, the binary components are effectively replaced by two rings of matter, which coincide if $m_{1}=m_{2}$. The density of each ring is proportional to the time spent by a component at each point. For a circular binary this ring is uniform and circular, and so the force field experienced by the third body is axisymmetric. Therefore the axial component of angular momentum of the third body (i.e., the component perpendicular to the plane of motion of the binary components) is conserved, and it follows, from overall angular momentum conservation, that the axial component of angular momentum of the binary is also conserved. It follows that the change in the magnitude of the angular momentum of the binary also vanishes (to first order). Since the change in semi-major axis over the entire encounter is exponentially small (Heggie 1975), it follows that the change in eccentricity also vanishes to first order. Note that this argument applies even without an expansion in powers of $a / r_{\mathrm{p}}$; it depends only on the use of averaging and the use of first-order perturbation theory (whereby it is assumed that the interaction is calculated as if the three bodies proceed on their unperturbed orbits.) Therefore it also follows that the octupole contribution would vanish to first order, and indeed all terms of the multipole expansion.

At second order it is not so clear what to expect. Averaging at first order ignores the fact that the third body causes the eccentricity of the binary to oscillate, and when this is taken into account (at second order) it is unclear whether the averaged mass distribution of the components is still axisymmetric. In their interpretation of their numerical results, Hut \& Paczyński (1984) considered that the change in eccentricity must indeed be a second-order effect. We have computed the second-order change in the eccentricity (via the change in the angular momentum), and find a non-zero result in general in the case $m_{1} \neq m_{2}$. This result is generated by the octupole term.

It turns out, however, that the distinction between first- and second-order effects is not an absolute one, but depends on the variables used in the computation. In the present paper we adopt the Lenz vector in preference to the angular momentum, as a means of computing the eccentricity. With this variable it turns out that a non-trivial result for the eccentricity induced in a circular binary is obtained at first order (provided again that $m_{1} \neq m_{2}$.) The result agrees with that obtained from a second-order computation of the change in angular momentum, and is also caused by the octupole interaction, but it is much more easily calculated using the Lenz vector, as only first-order perturbation theory is needed. It is not hard to see why second-order perturbation theory is required if the angular momentum is used. The angular momentum is proportional to $\sqrt{1-e^{2}}$, and so if the initial eccentricity is zero, the change in angular momentum is second order in the change of eccentricity.

Finally in this section we turn to the case of a binary with equal masses. Here the odd-order terms in the multipole expansion, equation (4), vanish, and it is easy to see that all first-order calculations, whether with the Lenz vector or the angular momentum, will yield a null result. Consider, for example, the method adopted in Appendix A. The perturbation is an odd function of $\mathbf{r}$ (the position vector of one component relative to the other), and it follows from equation (6) for the rate of change of the eccentric axis that this is also odd. A null result is obtained, therefore, if we average over the motion of the components. This result, which is so simple to prove at first order, also applies at all orders of perturbation theory, but the proof is somewhat technical, and is relegated to Appendix B. The implication of the result, however, is that the change in 
eccentricity must vanish more quickly (with increasing $r_{\mathrm{p}}$ ) than any power of the expansion parameter $a / r_{\mathrm{p}}$, and it is likely that the result vanishes exponentially as an inverse power of this parameter (cf. Section 2.3.2).

The problem of rigorously justifying the computation of exponentially small perturbations is one which has attracted considerable attention from mathematicians in recent years (e.g., Byatt-Smith \& Davie 1990). The difficulty is, of course, that standard perturbation methods proceed by expanding in powers of a small quantity, whereas these exponentially small results have no power series expansion. Unfortunately it still appears that each case must be treated separately if it is to be handled rigorously, and there is no general calculus for this class of problems. The present problem is certainly considerably more elaborate than those which have been treated properly. Our interest in this result is more pragmatic, however. We shall see that the result of Section 2.3.2, which exhibits exponential dependence, is important when it dominates the result from the octupole interaction (Section 2.3.1), and in this situation it does not require elaborate justification.

\subsection{Illustrations and Comparison with Numerical Results}

We have performed a large number of numerical integrations of encounters between a binary and a third star. In this section we present a sample of results, to establish the accuracy of our analytical expressions and to illustrate the results for some typical cases representative of the different possible regimes.

We shall focus primarily on parabolic encounters, which are particularly relevant astrophysically since the conditions in globular clusters are very close to the parabolic limit except for very wide binaries $(a \gg 1 \mathrm{AU})$. In general, in any star cluster, hyperbolic encounters are of importance only for binaries that are sufficiently wide to be classified as "soft" and therefore will tend to be easily disrupted by interactions (Hills 1975; Heggie 1975; Hut 1983). Deviations from the parabolic results remain small as long as the relative velocity at infinity for the encounter is less than the orbital velocity of the binary, and the encounter is not too distant (cf. eq. [1] and Section 3.2 below).

\subsubsection{Numerical Integrations}

The numerical integrations are fairly straightforward. We have used the Bulirsch-Stoer algorithm described in Press et al. (1992) to integrate the differential equations of motion of the three-body problem directly. Total energy and total angular momentum conservation is maintained typically to within $10^{-12}-10^{-11}$, for computations in double precision. This allows changes in eccentricity as small as $\delta e \sim 10^{-8}$ to be measured accurately. The input parameters for a given integration are the masses $m_{1}, m_{2}$ and $m_{3}$, the semi-major axis $a$, eccentricity $e$, and mean anomaly $\phi_{i}$ (or orbital phase, measured from pericentre) of the binary at $t=0$, the periastron distance $r_{\mathrm{p}}$ and velocity at infinity $V$ characterizing the outer orbit (cf. Section 2.1), the initial separation $r_{i}$ (distance between $m_{3}$ and the centre of mass of the binary at $t=0$ ), and the angles $\Omega, i$, and $\omega$ specifying the relative orientation of the two orbits (defined in Section 2.2). We generally adopt units such that $G=m_{3}=a=1$. Each integration follows the system through periastron passage and is continued until the final separation has increased to a value equal to the initial separation, $r_{f}=r_{i}$. Typically we use $r_{i} / r_{\mathrm{p}} \sim 10^{2}$. For a given set of input parameter values, we normally repeat the integrations for many different values of the initial phase $\phi_{i}$, covering systematically the interval from 0 to $2 \pi$. This allows us to compute a phase-averaged result and to study the extent of the phase dependence.

\subsubsection{Dependence on the Periastron Distance}

First we consider three simple situations where one of the three expressions (8), (11), and (13) dominates over the entire range of periastron separations where $\delta e$ remains small enough that a perturbative calculation applies.

In Fig. 1, we show the change in eccentricity of a binary with non-zero initial eccentricity, following an encounter with a star on a general inclined orbit. The three masses are assumed identical. The result is given by equation (8) to high accuracy. Here and in all subsequent figures the solid lines show the analytical results and the dots show the numerical results, with the "error bars" indicating the full extent of the dependence on orbital phase. In this case the phase dependence remains completely negligible down to very small values of $r_{\mathrm{p}} / a$. Notice that, as long as the three masses are comparable, the magnitude of the fractional change in eccentricity $|\delta e| / e$ predicted by equation (8) is always very small. Using equation (8) we see that a change $|\delta e| / e \gtrsim 1$ would require $m_{3} \gtrsim\left(r_{\mathrm{p}} / a\right)^{3} M_{12}$.

Now we turn to initially circular binaries. To isolate the octupole effect (equation [11]), we consider a binary with $m_{1} \neq m_{2}$ and we focus on distant encounters, with $r_{\mathrm{p}} / a \gtrsim 5$. The variation of the induced eccentricity $\delta e$ with $r_{\mathrm{p}}$ is shown in Fig. 2 for a coplanar, prograde encounter, and for two different values of $m_{2}$. Notice the steeper power-law behavior $\left(r_{\mathrm{p}}^{-5 / 2}\right)$ compared to the case of an initially non-circular binary $\left(r_{\mathrm{p}}^{-3 / 2}\right)$. Notice also the large drop in $\delta e$ when $m_{2}$ becomes nearly equal to $m_{1}$. To best illustrate the exponential regime (equation [13]), we show in Fig. 3 the variation of $\delta e$ in the equal-mass case. The induced eccentricity now drops much more rapidly, to values $\ll 10^{-7}$ for $r_{\mathrm{p}} / a \gtrsim 10$. Notice that equation (13) continues to predict $\delta e$ with remarkable accuracy down to very small values of $r_{\mathrm{p}} / a$.

In a general case, all three types of perturbations dominate in different ranges of $r_{\mathrm{p}}$. Consider for example a typical binary millisecond pulsar containing a neutron star of mass $m_{1}=1.4 M_{\odot}$ with a low-mass companion $m_{2}=0.2 M_{\odot}$ in a 


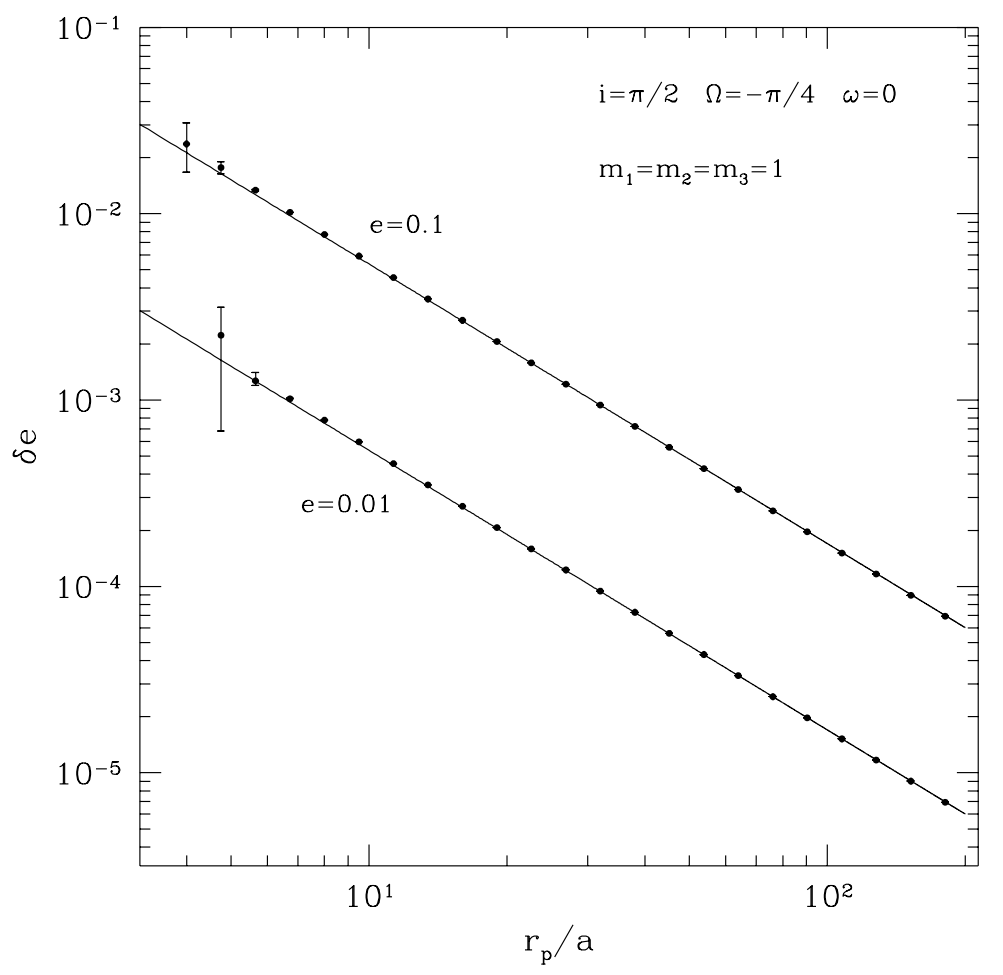

Figure 1. Change in eccentricity $\delta e$ for an initially non-circular binary (initial eccentricity $e=0.1$ or 0.01 ) perturbed by an encounter with a third star on an inclined, parabolic orbit with periastron distance $r_{\mathrm{p}}$ (given in units of the binary semi-major axis $a$ ). The dots show the results of numerical integrations; the lines show the analytical power-law result, equation (8). The "error bars" indicate the full extent of the phase dependence. For $r_{\mathrm{p}} / a \gtrsim 5$ this phase dependence is no longer visible.

nearly circular orbit of initial eccentricity $e=10^{-6}$; the binary is in the dense core of a globular cluster where the average stellar mass is $m_{3}=1 M_{\odot}$ (cf. Rasio \& Heggie 1995). Fig. 4 shows the final eccentricity $e_{f}=e+\delta e$ following a coplanar, prograde encounter, as a function of $r_{\mathrm{p}} / a$. The exponential regime corresponds to $r_{\mathrm{p}} / a \lesssim 5$. For $r_{\mathrm{p}} / a \gtrsim 200$, equation (8) dominates and the fractional change $\delta e / e$ is very small. In a wide range of intermediate values, $5 \lesssim r_{\mathrm{p}} / a \lesssim 200$, the octupole power-law result is dominant. As discussed in Rasio \& Heggie (1995), an important consequence of these results is that the cross section for inducing eccentricity in binary millisecond pulsars in clusters is much larger than was estimated in previous studies (see also section 3 below).

\subsubsection{Angular and Phase Dependences}

Although we have checked very carefully most of the phase and angular dependences appearing in our analytical expressions, we shall only present a small number of illustrative cases here. We focus on initially circular binaries, since the angular dependent factors appearing in equation (8), for the case of non-zero initial eccentricity, can be understood from elementary arguments, at least in part. We use the same typical values of the masses for a binary millisecond pulsar in a globular cluster as in Fig. 4, and we consider only parabolic encounters.

First, we look at the dependence on inclination for the induced eccentricity in the power-law regime. Fig. 5 shows the results for $r_{\mathrm{p}} / a=30$ and for two particular values of $\omega$. For $\omega=0$, the first term in the angular dependent factor of equation (11) vanishes and the dependence on inclination is $\propto\left|1-(5 / 4) \sin ^{2} i\right|$, giving zeros of $\delta e$ at $i \simeq 0.352 \pi$ and $i \simeq 0.648 \pi$. The maximum $\delta e$ is obtained for $i=0$ and $i=\pi$, with a secondary maximum at $i=\pi / 2$. For $\omega=\pi / 2$, the second term in the angular dependent factor vanishes and $\delta e \propto\left|\cos i\left[1-(15 / 4) \sin ^{2} i\right]\right|$, giving zeros at $i=\pi / 2, i \simeq 0.173 \pi$, and $i \simeq 0.827 \pi$. Again the maximum $\delta e$ is obtained for $i=0$ and $i=\pi$, with two secondary maxima at $i \simeq 0.335 \pi$ and $i \simeq 0.665 \pi$. The agreement between the numerical and analytical results is everywhere excellent, with deviations remaining always $\lesssim 3 \%$.

In the exponential regime, a much stronger phase-dependence is observed, and larger deviations are observed between the numerical and analytical results. This is not surprising, since the exponential regime corresponds to rather large values of the perturbation expansion parameter $a / r_{\mathrm{p}}$. In Fig. 6 , we illustrate the strong phase-dependence as well as the dependence on inclination for $r_{\mathrm{p}} / a=3.5$. The phase-dependence is largest for $i=0$ (coplanar prograde encounters), which also corresponds to the maximum value of $\delta e$ according to equation (13). For $i=\pi$, equation (13) predicts $\delta e=0$ even though the numerical result is clearly non-zero. The small discrepancies between numerical and analytical results in this regime come from neglecting 


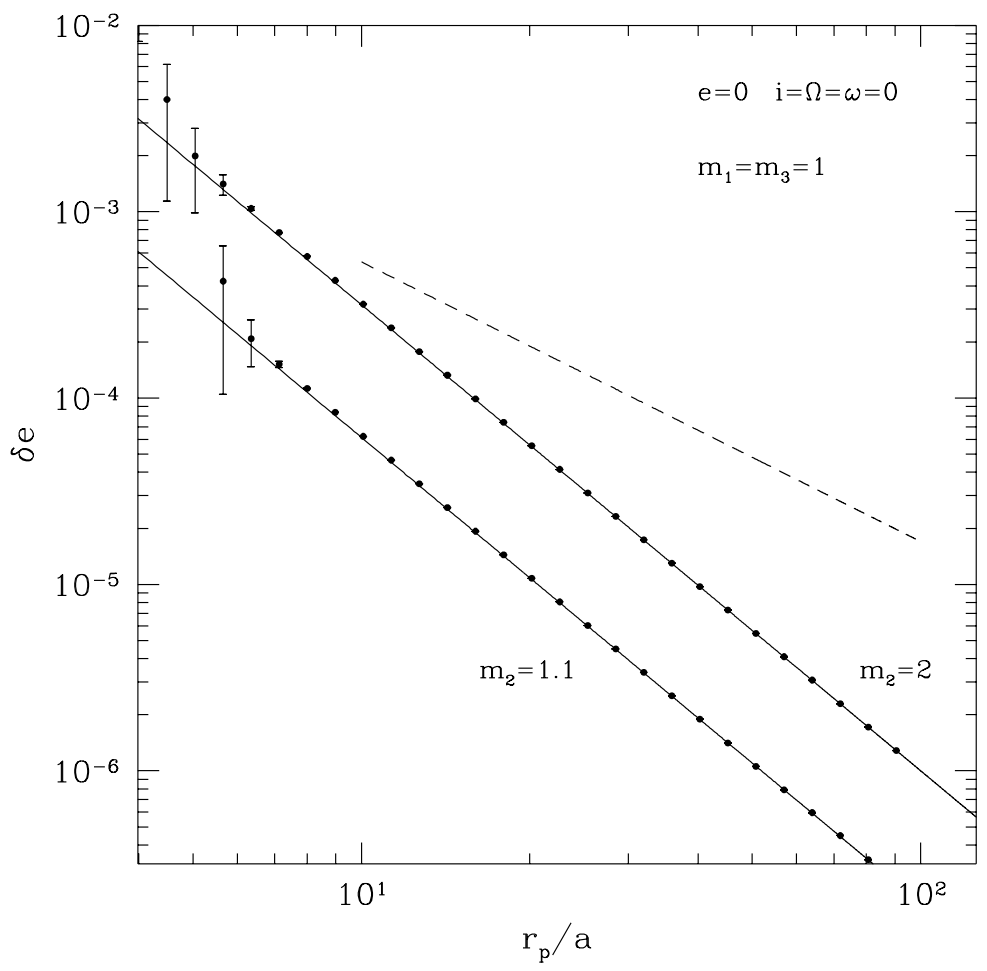

Figure 2. Change in eccentricity $\delta e$ for a binary with initial $e=0$ perturbed by an encounter with a third star on a coplanar, prograde, parabolic orbit with periastron distance $r_{\mathrm{p}}$ (given in units of the binary semi-major axis $a$ ). Conventions are as in Fig. 1 . The lines show the analytical result, equation (11), for two different values of $m_{2}$. The dashed line shows, for comparison, the variation of $\delta e$ for a non-circular binary with initial $e=0.01$ (same case as in Fig. 1).

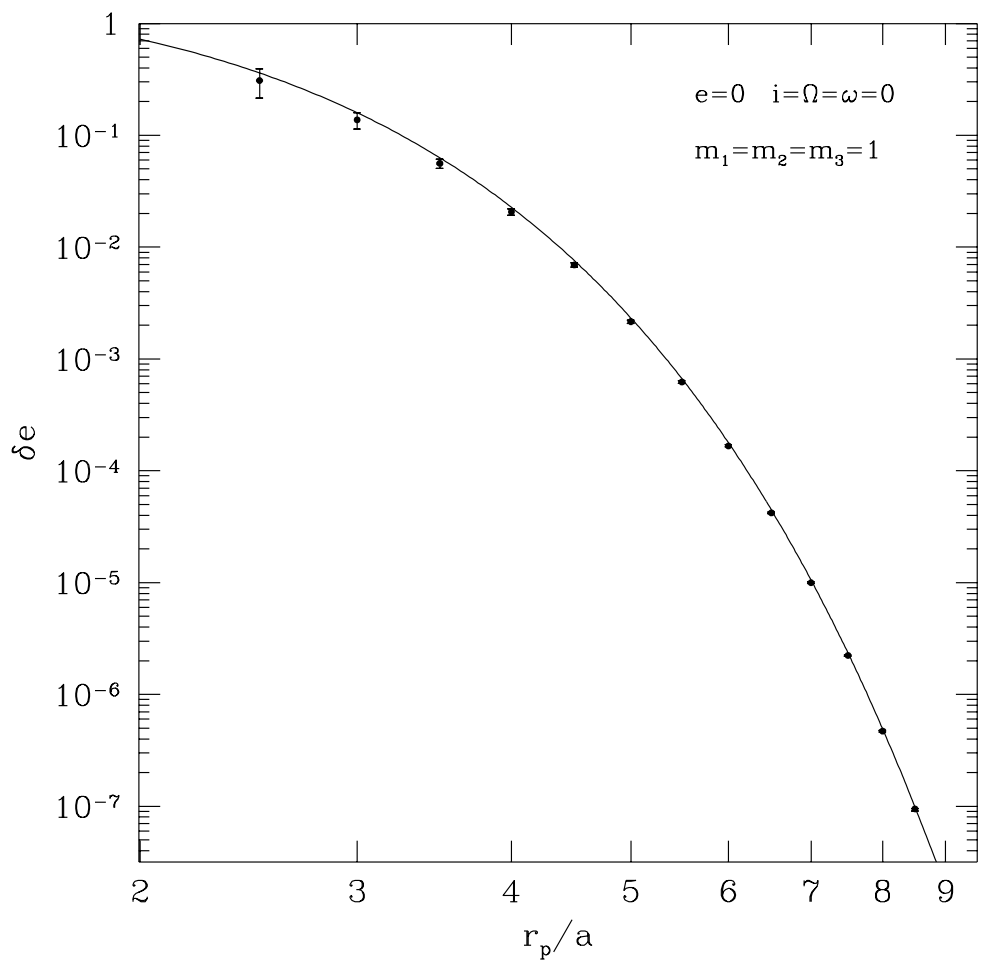

Figure 3. Change in eccentricity $\delta e$ for a binary with initial $e=0$ and containing two identical masses $m_{1}=m_{2}$. Conventions are as in Figs. 1 and 2. The line shows the analytical result, equation (13). 


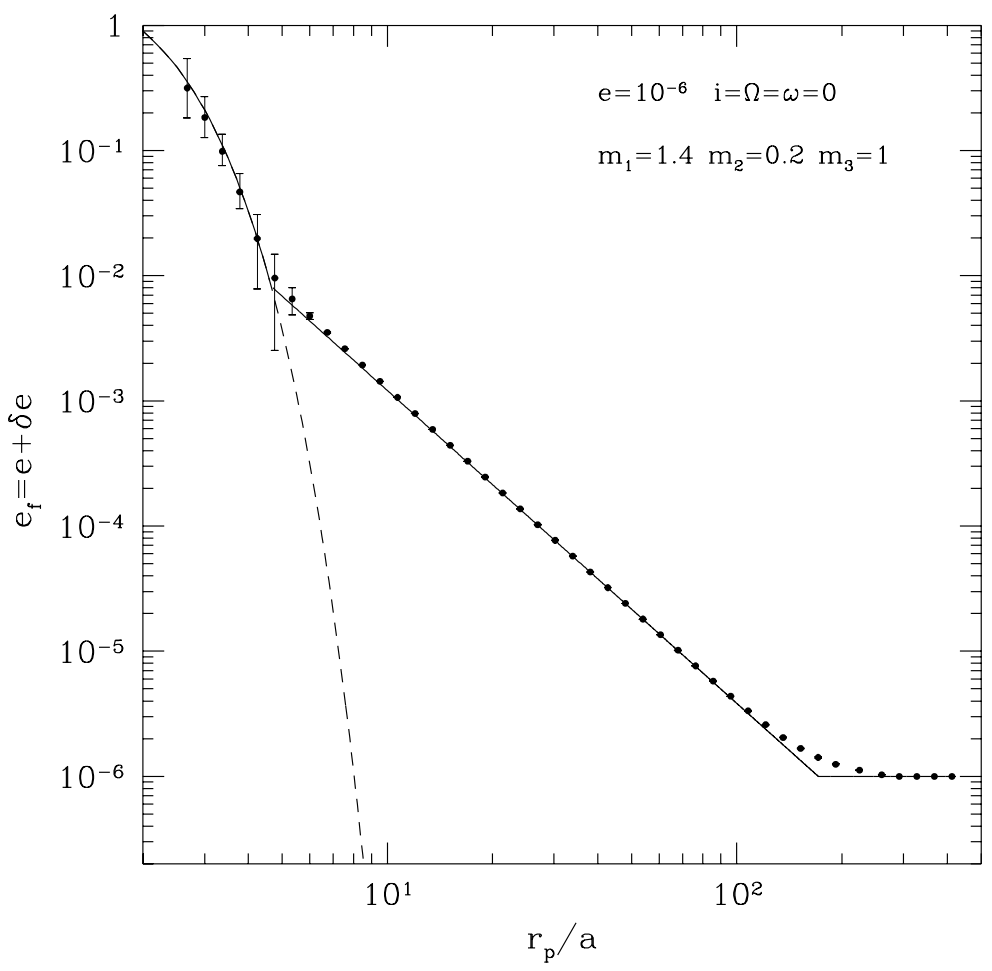

Figure 4. Final eccentricity $e_{f}=e+\delta e$ for a binary with initial $e=10^{-6}$ following a coplanar, prograde encounter. Conventions are as in Fig. 1. The masses have values representative of a typical binary millisecond pulsar in a globular cluster. The solid lines show the three separate analytical results, equations (8), (11), and (13). The dashed line shows the continuation of the exponential result, equation (13), for comparison. Notice that the octupole power-law result, equation (11), dominates over a wide range of intermediate values of $r_{\mathrm{p}}$.

the more rapidly oscillating term in the derivation of equation (13) (cf. Appendix A3), and from the octupole perturbations.

Other circumstances where a strong phase-dependence is observed are when two different perturbation effects contribute similar-amplitude changes in the eccentricity, as in Fig. 4 near $r_{p} / a \simeq 5$, or when $|\delta e| \simeq e$. As an example of the latter consider the case of a binary with $m_{1}=m_{2}$ and $i=0$ (so that both power-law expressions [8] and [11] vanish) and with a very small initial eccentricity $e=10^{-4}$, as illustrated in Fig. 7. The principal contribution to $\delta e$ is given by equation (13), though this was calculated for the case of an initially circular orbit. Since in the present case $e$ is small but non-zero, $\delta e$ must be interpreted as the magnitude of the vector $\delta \mathbf{e}$, and the final eccentricity is given by $e_{f}=|\mathbf{e}+\delta \mathbf{e}|$. Now the direction of $\delta \mathbf{e}$ depends on the phase of the binary, as can be seen by careful inspection of eq.(A21), and so if $|\delta \mathbf{e}|=e$ we see that $e_{f}=0$ for certain phases. In fact eq.(13) shows that the condition $|\delta \mathbf{e}|=e$ is satisfied when $r_{p} / a \simeq 6.2$. At this value the numerical result in Fig. 7 would have an "error bar" extending to arbitrarily small values of $e_{f}$ in this logarithmic plot. An alternative argument may be constructed by considering the time-reversal of such an encounter, i.e. one in which an initially circular binary is perturbed into an orbit with final eccentricity $e_{f}=10^{-4}$. Eq.(13) shows that this can only occur at the same critical value of $r_{p} / a$.

\subsubsection{Dependence on the Masses}

In general, when the three masses are all of comparable magnitude, and $m_{1} \neq m_{2}$, the mass-dependence of the induced eccentricity $\delta e$ is rather weak. Near $m_{1}=m_{2}$, however, the power-law expression, equation (11), can become arbitrarily small. The peculiar dependence of $\delta e$ on $\left|m_{1}-m_{2}\right|$ in the power-law regime is illustrated in Fig. 8 for a typical case. The agreement between analytical and numerical results is again excellent.

When $m_{3} \gg M_{12}$, as in the case of an encounter between a binary and a massive black hole, it is possible to find that the exponential result dominates even at rather large values of $r_{\mathrm{p}} / a$. This is because the duration of the encounter decreases as $m_{3}$ increases at fixed $r_{\mathrm{p}}$. In Fig. 9 we show the dependence of $\delta e$ on $m_{3}$ for fixed $r_{\mathrm{p}} / a=30$. The exponential result becomes dominant when $m_{3} \gtrsim 300$. For $m_{3} \gtrsim 3000$, the binary is disrupted by the encounter.

\subsubsection{Hyperbolic Encounters}

For fixed $r_{\mathrm{p}}$, as the relative velocity at infinity $V$ increases from zero, the duration of the encounter becomes shorter and shorter and we always expect the exponential result, equation (12), to dominate eventually. An example is shown in Fig. 10, 


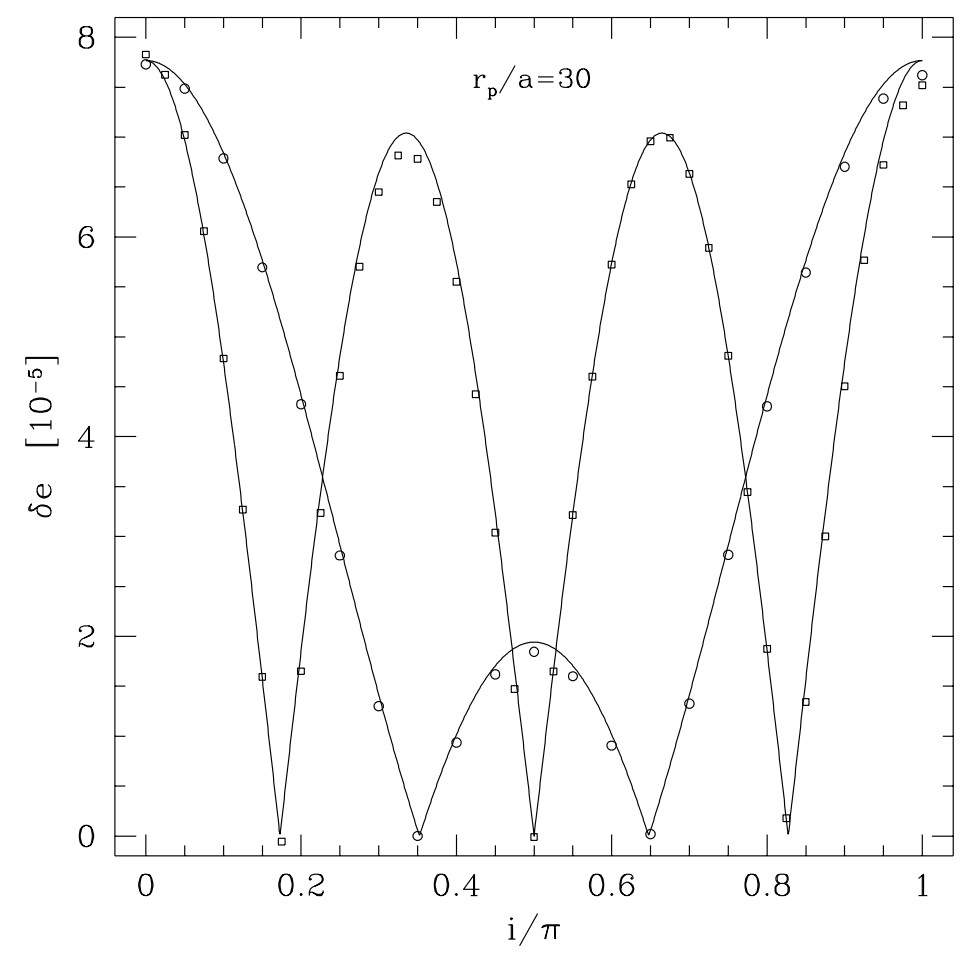

Figure 5. Dependence of induced eccentricity $\delta e$ on inclination for an initially circular binary with $m_{1}=1.4, m_{2}=0.2, m_{3}=1$, for a parabolic encounter with $r_{\mathrm{p}} / a=30$ (power-law regime). The round dots are for $\omega=0$, the square dots for $\omega=\pi / 2$. The lines show the analytical results, equation (11).

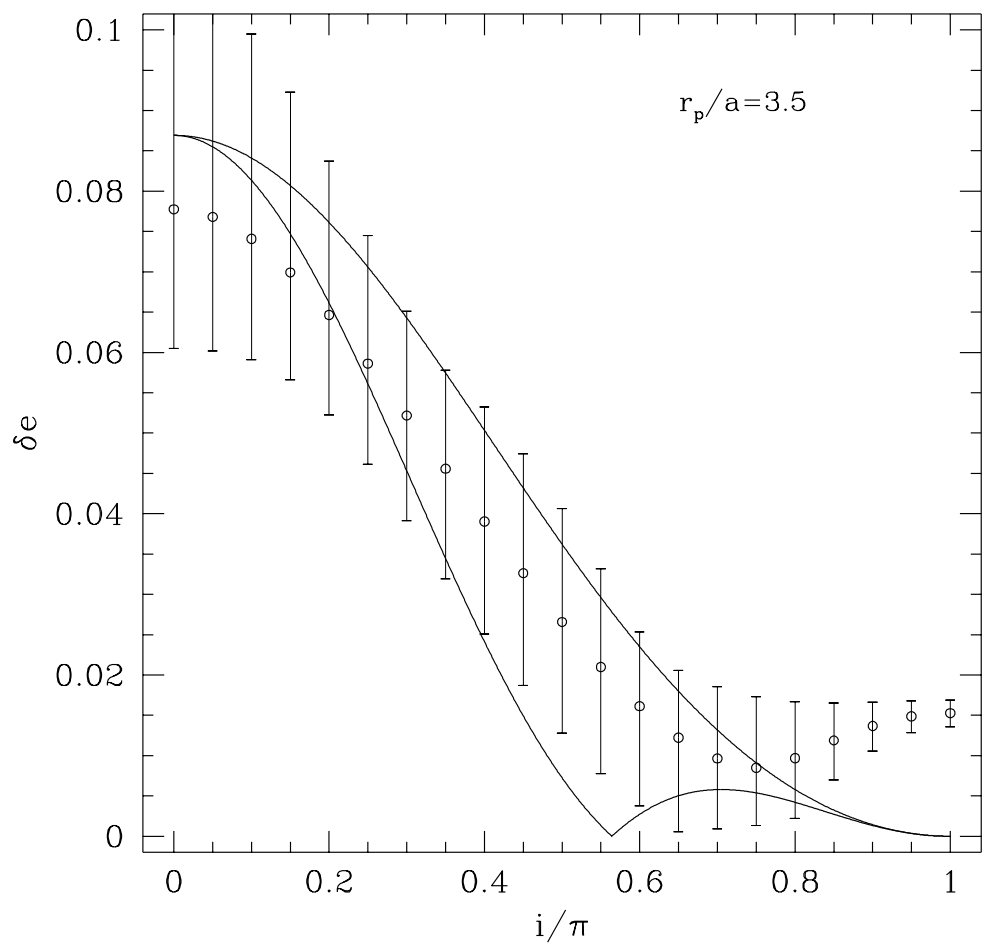

Figure 6. Same as Fig. 5 but for $r_{\mathrm{p}} / a=3.5$ (exponential regime). The two lines correspond to $\cos (4 \omega+2 \Omega)= \pm 1$ in equation $(13$ ), i.e., they show the extent of the theoretically predicted phase dependence. As before, the "error bars" show the full extent of the phase dependence in the numerical results while the dots show the phase-averaged numerical results. 


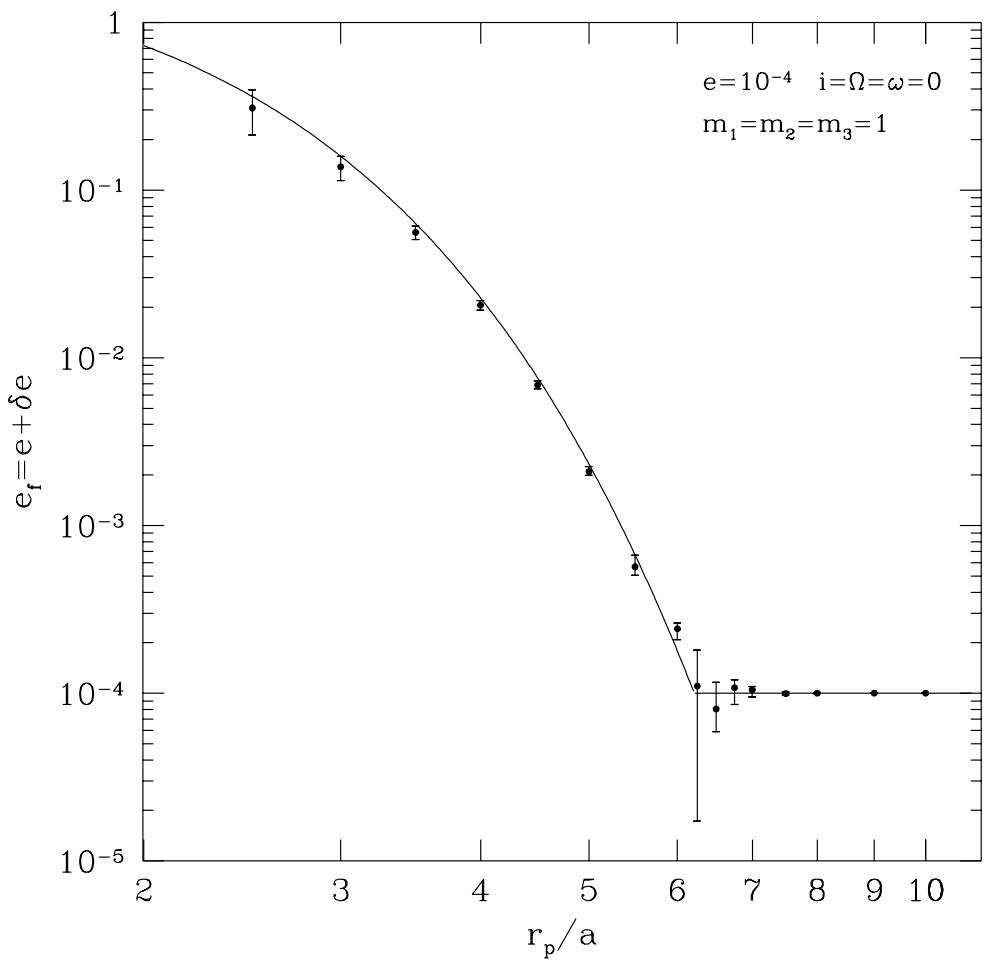

Figure 7. Same as Fig. 3 but for a binary with non-zero initial $e=10^{-4}$. Notice the strong phase dependence near $r_{\mathrm{p}} / a \simeq 6-7$, where the magnitude of $\delta \mathbf{e}$ (given by eq. [13]) is comparable with the initial value of $e$.

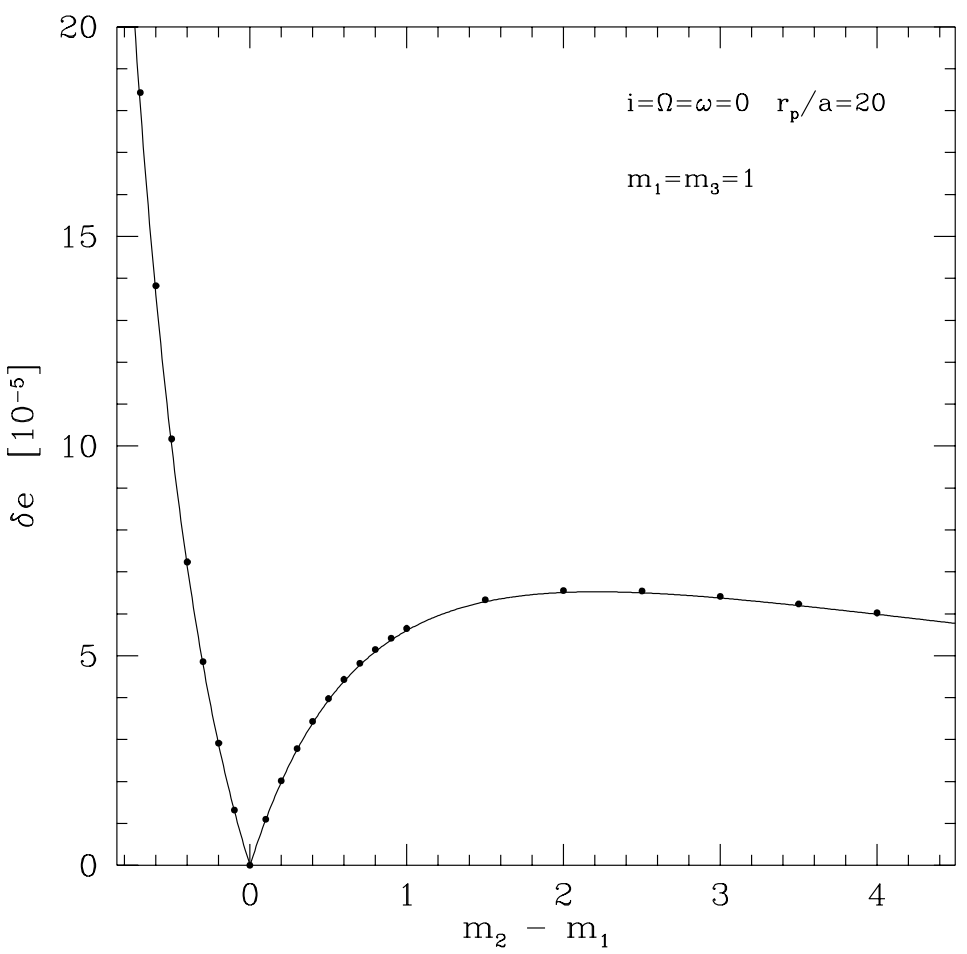

Figure 8. Dependence of $\delta e$ on the masses near $m_{1}=m_{2}$ for the power-law regime. Equation (11) predicts $\delta e=0$ for $m_{1}=m_{2}$, in agreement with the numerical results.

where we fix $r_{\mathrm{p}} / a=30$. Although the secular octupole effect is completely dominant in the parabolic limit, non-secular effects become dominant for $V /\left(G m_{3} / a\right)^{1 / 2} \gtrsim 4$, or $V \gtrsim 120 \mathrm{~km} \mathrm{~s}^{-1}\left(m_{3} / 1 M_{\odot}\right)^{1 / 2}(a / 1 \mathrm{AU})^{-1 / 2}$. Between $V=0$ and this value, $\delta e$ 


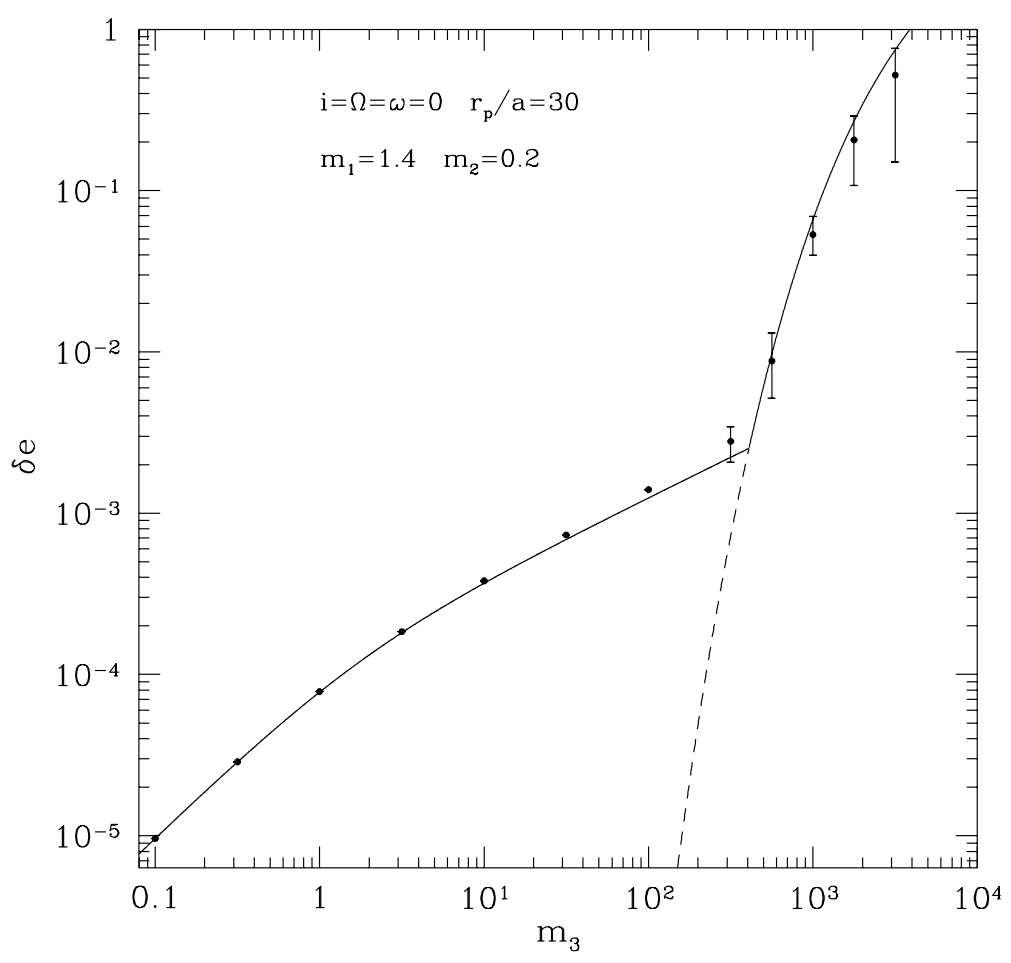

Figure 9. Same as Fig. 4 but showing the dependence of $\delta e$ on $m_{3}$. For sufficiently large $m_{3}$ (here for $m_{3} \gtrsim 300$ ), non-secular effects can always become dominant, even for encounters with large $r_{\mathrm{p}} / a$.

decreases by almost an order of magnitude, but then increases again as $V$ increases in the exponential regime. In contrast, Fig. 11 shows a case where the exponential result dominates everywhere, which typically happens at sufficiently small $r_{\mathrm{p}}$. Here $\delta e$ increases at first, reaches a maximum at $V /\left(G m_{3} / a\right)^{1 / 2} \simeq 2$ and then decreases with increasing $V$. Note that the impulsive limit, where $\delta e \propto V^{-1}$ (cf. Section 3.2 and Appendix A4), would only be reached for much higher values of $V$, and therefore does not seem astrophysically relevant.

\section{CROSS SECTIONS FOR ECCENTRICITY CHANGE}

\subsection{Analytical Results}

While the results of Section 2 are fully detailed, giving the dependence on all the circumstances of the encounter, for the purpose of applications it is necessary to average over several parameters which are normally only known statistically. In the present section we subject the results to some further processing in order to extract the cross section for encounters leading to changes in eccentricity above a given value. The cross section is essentially the square of the impact parameter $p$, but we shall restrict attention to the case of near-parabolic encounters. In this case the relation between $p$ and the distance of closest approach reduces to

$r_{\mathrm{p}} \simeq \frac{p^{2} V^{2}}{2 G M_{123}}$

\subsubsection{Binaries with Non-Zero Initial Eccentricity}

Consider first binaries with non-zero initial eccentricity. From equation (14) it follows that the dependence of equation (8) on the circumstances of the encounter may be summarised as

$\delta e=-A p^{-3} \sin 2 \Omega \sin ^{2} i$,

where

$A=\frac{15 \pi}{4} \frac{m_{3} M_{123}}{\sqrt{M_{12}}} \frac{(G a)^{3 / 2}}{V^{3}} e \sqrt{1-e^{2}}$.

Now we compute the cross section for events in which $\delta e>\delta e_{0}$, where $\delta e_{0}$ is some value of interest. We shall suppose that $\delta e_{0}>0$, and then it will be obvious how to extend the result to negative values, as expression (8) obviously takes positive 


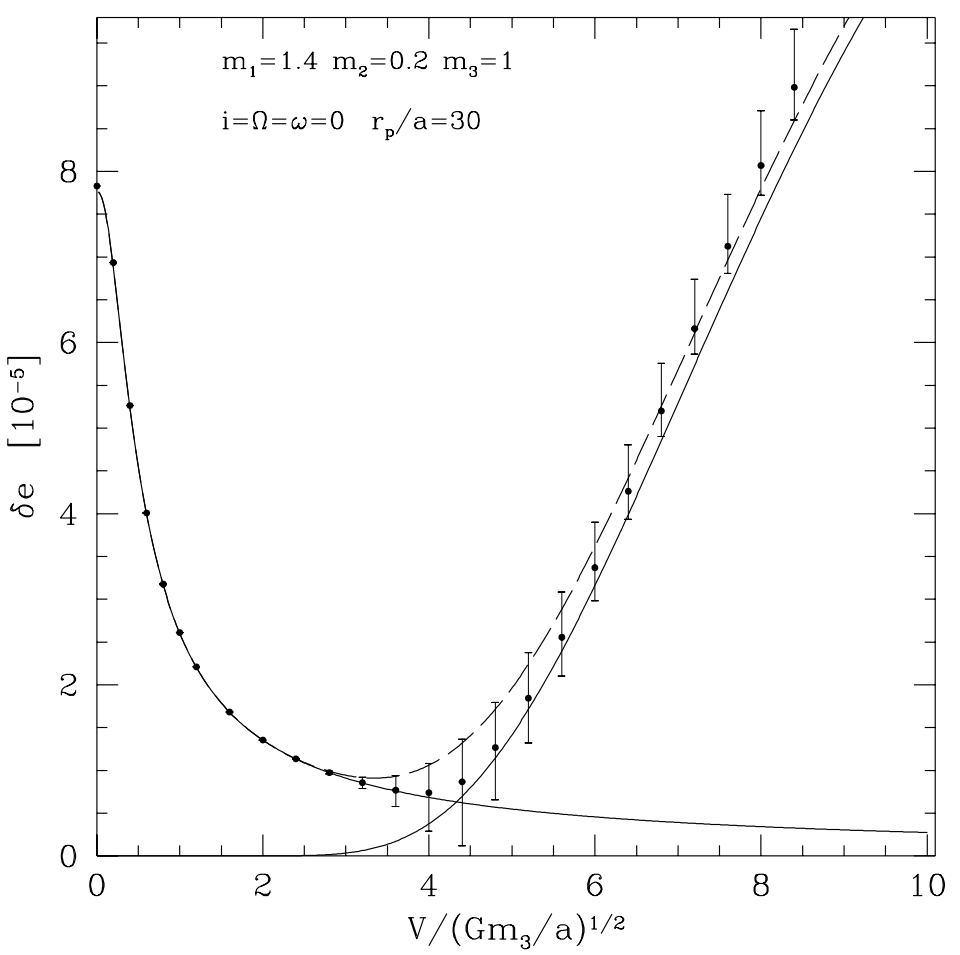

Figure 10. Variation of the induced eccentricity $\delta e$ as a function of $V$, the relative velocity at infinity for an encounter with fixed $r_{\mathrm{p}} / a=30$. The two solid lines show the power-law and exponential analytical results (equations [9] and [12]); the dashed line shows their sum. The exponential result dominates for $V /\left(G m_{3} / a\right)^{1 / 2} \gtrsim 4$.

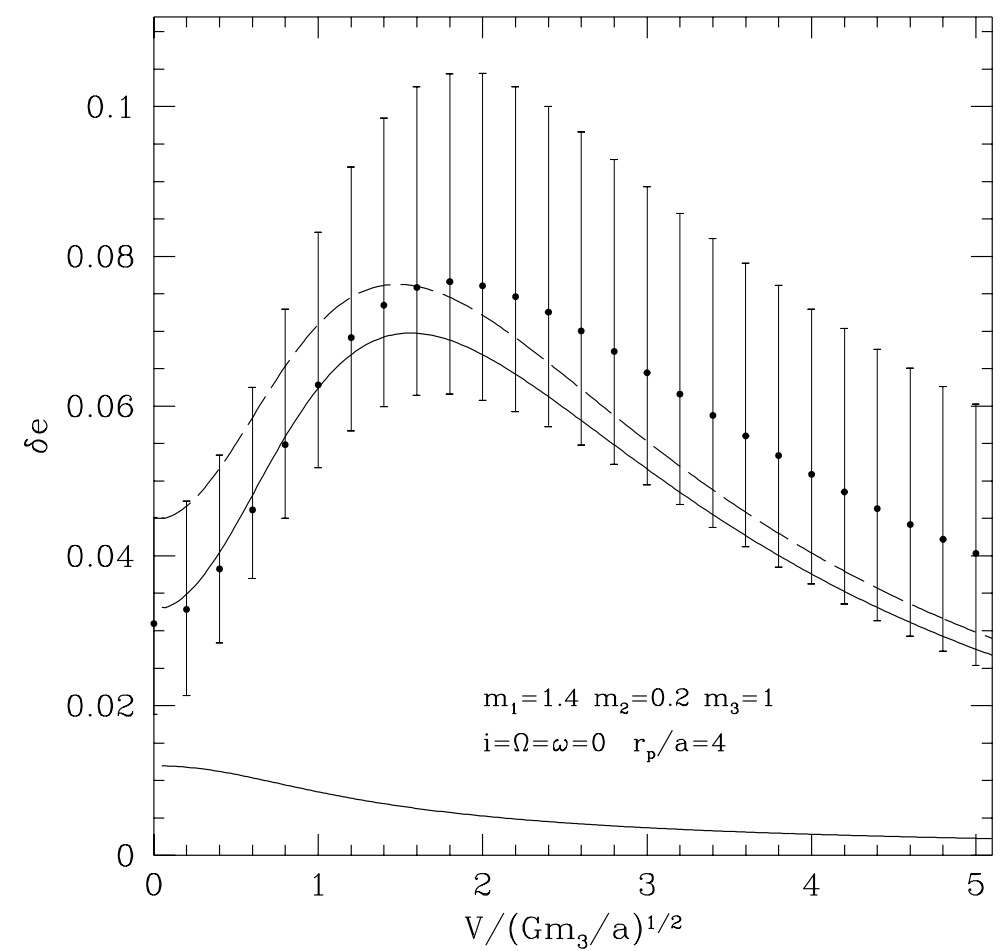

Figure 11. Same as Fig. 10 but for $r_{\mathrm{p}} / a=4$. The exponential result (upper solid line) dominates everywhere in this case. Notice how the variation of $\delta e$ is opposite to that shown in Fig. 10, with $\delta e$ increasing at first, reaching a maximum for $V /\left(G m_{3} / a\right)^{1 / 2} \simeq 2$, then decreasing at higher velocities. 
Table 1. Correction to the Asymptotic Cross Section in the Exponential Regime

$\begin{array}{llllllllll}M_{123} \delta e_{0} \\ \frac{3 \sqrt{6 \pi} m_{3}}{\chi} & 0.333 & 0.25 & 0.2 & 0.1 & 0.033 & 0.01 & 0.001 & 0.0001 & 0.00001 \\ & 0.112 & 0.228 & 0.307 & 0.497 & 0.705 & 0.850 & 0.938 & 0.968 & 0.982\end{array}$

and negative values with equal probability. Using the appropriate statistical distributions of $i$ and $\Omega$, we write

$\sigma\left(\delta e>\delta e_{0}\right)=\int 2 \pi p d p \frac{1}{2 \pi} d \Omega \frac{1}{2} \sin i d i$

where the domain of integration is given by $p^{3}<-A \sin 2 \Omega \sin ^{2} i, 0<i<\pi$ and, for $\Omega$, the union of the intervals $[\pi / 2, \pi]$ and $[3 \pi / 2,2 \pi]$ (to ensure that $\delta e$ has the same sign as $\delta e_{0}$ ). The integrations are straightforward, and lead to the result

$\sigma\left(\delta e>\delta e_{0}\right)=\left(\frac{A}{\delta e_{0}}\right)^{2 / 3} \frac{9 \sqrt{ } 3}{14 \pi}\left[\Gamma\left(\frac{2}{3}\right) \Gamma\left(\frac{5}{6}\right)\right]^{2}$.

After the definition of $A$ is reinstated the result becomes

$\sigma\left(\delta e>\delta e_{0}\right)=\frac{9 \sqrt{ } 3}{14 \pi}\left(\frac{15 \pi}{4}\right)^{2 / 3}\left[\Gamma\left(\frac{2}{3}\right) \Gamma\left(\frac{5}{6}\right)\right]^{2}\left(\frac{m_{3}^{2}}{M_{12} M_{123}}\right)^{1 / 3} \frac{G M_{123} a}{V^{2}} e^{2 / 3}\left(1-e^{2}\right)^{1 / 3} \delta e_{0}^{-2 / 3}$.

It is clear that the cross section for negative changes, i.e. $\delta e<-\delta e_{0}$, where $\delta e_{0}>0$, is given by the same expression.

\subsubsection{Initially Circular Binaries}

Now we turn to the cross section corresponding to the parabolic result for a circular binary in the power-law regime, equation (11). Everything is as straightforward as in the previous case, with some minor alterations. First, only positive values of $\delta e_{0}$ are relevant. Second, the appropriate distribution for $\omega$ is uniform on the range $[0,2 \pi]$. Third, the integration cannot apparently be performed analytically, and it is a tricky job numerically because the integrand is not smooth at certain points in the relevant domain. For this reason we have opted for a Monte Carlo evaluation, and so we find that

$\sigma\left(\delta e>\delta e_{0}\right) \simeq 4.62\left(\frac{m_{3}\left|m_{1}-m_{2}\right|}{M_{12}^{2}}\right)^{2 / 5}\left(\frac{M_{12}}{M_{123}}\right)^{1 / 5} \frac{G M_{123} a}{V^{2}} \delta e_{0}^{-2 / 5}$.

The coefficient is correct to the stated number of figures.

The exponential regime for circular binaries is somewhat trickier, because the dependence on $r_{\mathrm{p}}$ in equation (13) is not a simple power law. Using equation (14) we first recast equation (13) as

$\delta e=A\left(B p^{3}\right)^{1 / 2} \exp \left(-B p^{3}\right) f_{2}(i, \omega, \Omega)$,

where

$A=3 \sqrt{6 \pi} m_{3} / M_{123}, \quad B=\frac{1}{3}\left(\frac{M_{12}}{M_{123}}\right)^{1 / 2} \frac{V^{3}}{\left(G M_{123} a\right)^{3 / 2}}$,

and $f_{2}$ is the geometric factor at the end of equation (13). Proceeding as in the previous cases, we can write the desired cross section as

$\sigma\left(\delta e>\delta e_{0}\right)=\int 2 \pi p d p \frac{1}{2} \sin i d i \frac{1}{2 \pi} d \theta$,

where we have set $\theta=4 \omega+2 \Omega$ : it is clear that the appropriate distribution is uniform. The integration with respect to $p$ gives a factor $\int p d p=p^{2} / 2$, where $p$ is the root of equation (21) with $\delta e$ replaced by $\delta e_{0}$. It follows that

$\sigma\left(\delta e>\delta e_{0}\right)=\frac{1}{4 B^{2 / 3}} \int x^{2 / 3} \sin i d i d \theta$

where $x$ is the larger root of $x-(1 / 2) \ln x=\ln \left(A f_{2} / \delta e_{0}\right)$. An approximate solution is $x=\ln \left(A / \delta e_{0}\right)$, which is valid when $\ln \left(A / \delta e_{0}\right) \gg 1$, and in that limit we have $\sigma\left(\delta e>\delta e_{0}\right)=\pi\left[B^{-1} \ln \left(A / \delta e_{0}\right)\right]^{2 / 3}$, i.e.

$\sigma\left(\delta e>\delta e_{0}\right)=\pi\left(\frac{9 M_{123}}{M_{12}}\right)^{1 / 3} \frac{G M_{123} a}{V^{2}}\left[\ln \left(\frac{3 \sqrt{6 \pi} m_{3}}{M_{123} \delta e_{0}}\right)\right]^{2 / 3} \chi\left(\frac{\delta e_{0}}{A}\right)$.

Here $\chi$ is a correction factor which will be used to allow for a range of values of $\delta e_{0} / A$; in the limit of $\delta e_{0} \ll A$ we use $\chi(0)=$ 1. For other values of $\delta e_{0} / A$ we have evaluated the integral in equation (24) by a Monte Carlo technique, with results which are given in Table 1 as the correction $\chi$ to the foregoing asymptotic result. The data are accurate to 1 unit in the last digit printed.

The maximum possible value of $\delta e_{0}$ is $A / \sqrt{2 e}, \simeq 5.6 m_{3} / M_{123}$. 


\subsection{Comparison with Previous Work}

It is interesting to compare these cross sections with those published by other authors.

Hut \& Paczyński (1984) gave a result which may be expressed, using our notation, as

$\sigma_{H P}\left(\delta e>\delta e_{0}\right)=(4.7 \pm 0.9) \frac{G M_{123} a}{V^{2}} \frac{\sqrt{m_{3} M_{12}}}{M_{123}} \delta e_{0}^{-1 / 3}$.

This was obtained by numerical scattering experiments for hard, initially circular binaries, and so we shall compare it with our equation (20). The result is

$\frac{\sigma_{H P}}{\sigma_{H R}}=(1.02 \pm 0.19) \frac{m_{3}^{0.1} M_{12}^{1.1}}{\left|m_{1}-m_{2}\right|^{0.4} M_{123}^{0.8}} \delta e_{0}^{1 / 15}$.

According to Hut \& Paczyński (1984), their result is valid to within the stated accuracy of $20 \%$ for certain ranges of $a$ and $V$, and for mass ratios in the ranges $0.03<m_{2} / m_{1}<0.3,0.2<m_{3} / m_{1}<1$. In this range of masses, the mass-dependent factor in equation (27) varies steadily from about 0.59 (at $\left.m_{2}=0.03 m_{1}, m_{3}=m_{1}\right)$ to about $0.95\left(\right.$ at $\left.m_{2}=0.3 m_{1}, m_{3}=0.2 m_{1}\right)$. Actually for the most discrepant result here the scattering cross section of Hut \& Paczyński applies to binaries which include the borderline between hard and soft pairs, because $m_{2}$ is so low. Therefore it is probable that their cross section is a satisfactory compromise in this domain. Certainly, our own result is not intended to be applicable to such encounters.

Now we turn to a detailed comparison with a representative result from the paper by Rappaport et al. (1989, hereafter RPV). They give results for a single-parameter family of circular binaries in each of two environments, characterised by mixtures of stars with given mass, number density and velocity dispersion. We have chosen to compare with their result for the hardest binary (one of orbital period 3 days) in their model for the stellar population of the star cluster $\omega$ Cen. Our first step is to average our result, equation (20), over a Maxwellian distribution of velocities, though with an extra factor of $V$ in the averaging to account for the enhanced rate of interaction between stars of high relative speed (as was done by $\mathrm{RPV})$. The result is that the factor $V^{2}$ in equation (20) is replaced by $2\left(\left\langle v_{b}^{2}\right\rangle+\left\langle v_{3}^{2}\right\rangle\right) / 3$, where the averages are the mean square three-dimensional speeds of the binary and the third body, respectively. Next the cross section is averaged over the stellar species in the mixture specified by RPV. Then a similar process is carried out for the cross section, equation (25), corresponding to the exponential regime. This is slightly more awkward, as the averaging over the stellar species must be carried out afresh for each value of $\delta e_{0}$. For comparison with the results of RPV we simply adopt the larger of our two cross sections at each value of $\delta e_{0}$.

Fig. 12 shows the ratio of RPV's cross section to ours as a function of $\delta e_{0}$. The change in slope near $\delta e_{0} \simeq 0.003$ is associated with the point at which the cross section of equation (25) begins to dominate that of equation (20). The change at $\delta e_{0}=0.01$ has a similar origin in the work of RPV: the functional form of their cross section changes at this point. From there up to the point where $\delta e_{0} \simeq 0.5$ our cross section is systematically higher, by about $60 \%$, than that of RPV. The reason for this systematic disagreement is not certain, but it is clear that our approach of using the maximum cross section at each value of $\delta e_{0}$ is rather crude. The sharp drop towards 0 as $\delta e_{0} \rightarrow 1$ reflects the fact that our cross section does not vanish in this limit, whereas that of RPV does so. Turning now to very small values of $\delta e_{0}$, we see that the cross section of RPV decreases monotonically relative to ours. Indeed, their expressions are similar to our equation (25), valid in the exponential regime but not in the power-law regime. This may be related to their use of relatively short and low-precision numerical integrations, which are probably inadequate for computing the very small changes in eccentricity corresponding to the power-law regime. It must also be pointed out, however, that our cross sections were calculated for parabolic encounters, which is inappropriate when $r_{\mathrm{p}} / a \gtrsim G M_{123} /\left(a V^{2}\right)$. Substitution of typical values into equation (11) therefore shows that the validity of our result is restricted to the range $\delta e_{0} \gtrsim 10^{-5}$ in this case.

Next we turn to the work of Hills (1991), who computed cross sections for equal-mass binaries in encounters with more massive intruders $\left(m_{3} \gg m_{1}=m_{2}\right)$. The initial eccentricity was $e=10^{-4}$, and since Hills' results correspond to much larger changes, in the range $\delta e>0.1$, we shall compare equation (25) with his results extrapolated to $V=0$. He gives a scaled cross section $\sigma(\delta e>0.3)$ for mass ratios in the range $10<m_{3} / m_{1}<10^{4}$. Our results show the same trend with increasing mass ratio, but are consistently smaller by about 10-20\%. The dependence of the cross section on $\delta e_{0}$ can also be checked, as Hills gives the ratios of $\sigma\left(\delta e>\delta e_{0}\right)$ for the values $\delta e_{0}=0.1,0.3$ and 0.7 . For the smaller values of $\delta e_{0}$ our result agrees with Hills' to within the same accuracy of $10-20 \%$. For the larger values the agreement is poorer: Hills finds values of $\sigma(\delta e>0.3) / \sigma(\delta e>0.7)$ in the range 1.5-3, whereas our results yield a ratio closer to 1.4 . Since our results are based on assumptions which are valid for small eccentricity changes, it is perhaps surprising that the agreement is so good.

Finally we compare our results with those of Phinney (1992), who gives formulae for the induced eccentricity in an initially circular binary, and an approximate cross section. Like us, Phinney assumes that $r_{\mathrm{p}} \gg a$. For simplicity, he only considers the coplanar case and he assumes a straight-line (i.e., extremely hyperbolic) trajectory. In this limiting case, the exponential result always dominates and the appropriate comparison is to our equation (12) in the limit where $e^{\prime} \rightarrow \infty$ and for $i=0$. Identifying Phinney's quantity $\lambda$ with $\left(M_{12} / M_{123}\right)^{1 / 2}\left(r_{p} / a\right)^{3 / 2}\left(e^{\prime}\right)^{-1 / 2}$ in our notations, we find perfect agreement with Phinney's equation (5.2). Note that Phinney's equations (5.2) and (5.3) have been mislabelled: they correspond to the 


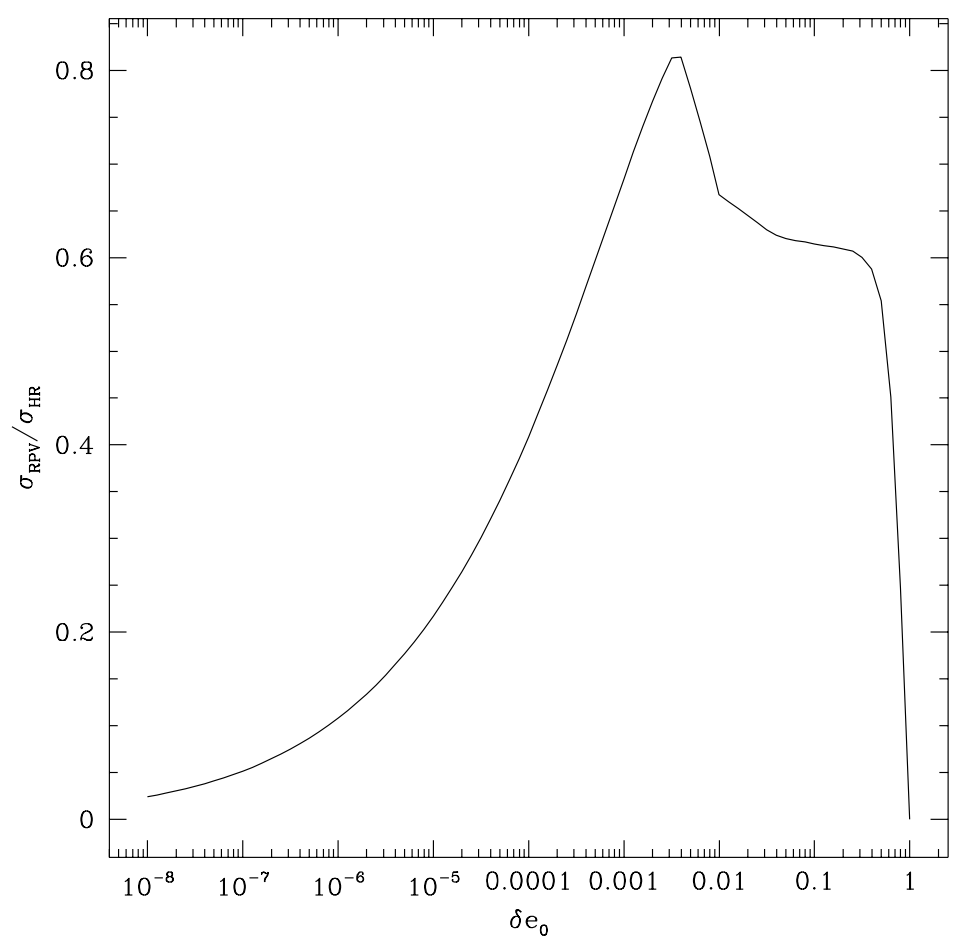

Figure 12. Comparison between the scattering cross sections $\sigma\left(\delta e>\delta e_{0}\right)$. The result of Rappaport et al. (1989), denoted by the subscript RPV, is compared with the cross section derived from the results of this paper, for a range of values of $\delta e_{0}$.

prograde and retrograde cases, respectively (Phinney, personal communication). Phinney's result for the coplanar retrograde case is of higher order and was neglected in our derivation of equation (12).

Phinney (1992) also gives a result for fast encounters (the "impulsive limit"), which we now consider briefly, although we do not expect this case to be of importance in most astrophysical applications (one possible exception being the perturbation of wide circular binaries by halo stars in the Galaxy). We give a derivation in Appendix A4, and our result for coplanar encounters with an initially circular binary is

$\delta e=\frac{2 G^{1 / 2} m_{3} a^{3 / 2}}{M_{12}^{1 / 2} V r_{\mathrm{p}}^{2}}|\cos \theta|\left(\cos ^{2} \theta+4 \sin ^{2} \theta\right)^{1 / 2}$,

where $\theta$ is the angle between the vectors $\mathbf{r}$ and $\mathbf{R}$ at the time of pericentre passage. This result vanishes when $\theta=90^{\circ}$, which can be understood because in that case the third body generates equal impulses in both components, and therefore does not alter their relative motion. Phinney's equation (5.1) as published differs in the trigonometrical factor. This is because of a typographical error: the first term inside the square root, which is printed as $3 \sin ^{2} \theta_{0}$ should have been $3 \sin ^{2} 2 \theta_{0}$ (Phinney, personal communication). With this typo corrected, it is easy to show that Phinney's equation (5.1) is in fact identical to our equation (28).

\section{CONCLUSIONS AND DISCUSSION}

We now summarise our main results. They describe the change $\delta e$ in the eccentricity of a binary of semi-major axis $a$ which experiences an encounter with a third body, in the following regime: if $r_{\mathrm{p}}$ is the distance of closest approach of the perturber, we suppose that $r_{\mathrm{p}}$ considerably exceeds $a$ and that the time scale of the encounter considerably exceeds the period of the binary. With these assumptions our results are of most relevance to hard binaries, and the orbit of the third body is nearly parabolic.

1. When the induced change in eccentricity, $\delta e$, is small compared with the initial eccentricity of the binary, then $\delta e$ falls off as $\left(a / r_{\mathrm{p}}\right)^{3 / 2}$. The relevant results are equation (7) for a hyperbolic encounter and equation (8) in the parabolic limit. Other factors affecting the result are the masses of the participants, the geometry of the encounter, the initial eccentricity of the binary and the orbit of the perturber. The result comes from the quadrupole interaction between the binary and the third body. Its main relevance is to binaries with a large initial eccentricity (Fig. 1).

2. When $\delta e$ is large compared with the initial eccentricity, and $r_{\mathrm{p}}$ much exceeds $a$, the final eccentricity falls off in general as $\left(a / r_{\mathrm{p}}\right)^{5 / 2}$ (equations [9] and [11]), and also depends on various other factors. The fact that this result originates in the 
octupole interaction accounts for the extra power. This result is of relevance mainly to binaries whose initial eccentricity is small (e.g., initially circular binaries), except for very close encounters (cf. Figs. 2 and 4).

3. When $r_{\mathrm{p}}$ exceeds $a$, but not by a large factor, the induced eccentricity falls off exponentially with a power of $r_{\mathrm{p}} / a$, though the coefficient also depends on this ratio, and other factors (equations [12] and [13]). The exponential factor occurs because, at these values of $r_{\mathrm{p}} / a$, the time scale of the encounter begins to be comparable with the period of the binary. It is mainly of relevance to binaries with small initial eccentricity, and to encounters which lead to a large induced eccentricity, but at periastron distances $r_{\mathrm{p}}$ where the quadrupole interaction is still dominant. It is also relevant to the very special case of a binary with components of equal mass (Fig. 3), or to the more important case of a massive perturber (Fig.9).

4. Cross sections corresponding to these three results are given in equations (19), (20) and (25). The first two depend on certain powers of the change in eccentricity, while the dependence in the third is approximately logarithmic.

These results have been compared with numerical data in various ways. We have tested the predicted dependence of the induced eccentricity change $\delta e$ on the encounter distance $r_{\mathrm{p}}$ (Figs. 3, 4 and 7), on the masses of the stars (Figs. 8 and 9), and on the geometry of the encounter (Figs. 5, 6, 10 and 11). We have almost always found excellent agreement between numerical and analytical results.

We have also compared our results, and especially the cross sections, with those derived by other authors. The main point at which previous work requires revision is the behaviour of initially circular binaries. Previously it was thought that the induced eccentricity $\delta e$ falls off exponentially with encounter distance, but it is now clear that this result applies only to a limited range of periastron distance, beyond which $\delta e$ falls off only as a power of the distance. The effect of this is that the cross section for encounters leading to small (but observable) induced eccentricities is very considerably larger than was previously sometimes thought.

The advantage of using our formulae for the induced eccentricity change in future investigations is speed. Where the parabolic approximation is valid, the stated cross sections may be used directly, and it is a straightforward matter to compute the cross section for any reasonable mass spectrum. Where the parabolic approximation is invalid, but the encounters are still both slow and tidal, cross sections may be computed in a Monte Carlo fashion, the outcome of each encounter being obtained from the appropriate formulae of Section 2. In this way estimates of cross sections in a wide variety of collisional stellar systems may be estimated much more rapidly than in the past, without the need for numerous careful and time-consuming three-body integrations.

Currently the most important application of our results concerns low-mass binary millisecond pulsars (LMBPs) in globular clusters. These are formed when an old neutron star in a binary system accretes material from a red-giant companion. This leads to the production of a spun-up (recycled) pulsar with a low-mass white-dwarf companion in a wide, circular orbit (typical eccentricities at birth being in the range $e_{i} \sim 10^{-6}-10^{-3}$ ). This formation process is well understood theoretically (Verbunt 1993; Phinney \& Kulkarni 1994). In globular clusters, the wide orbits of LMBPs can be perturbed significantly by passing stars. Thus final eccentricities $e_{f} \gg e_{i}$ can be observed today, and the measured values contain important information about the dynamical history of the binaries and their environment. In fact Rasio \& Heggie (1995) have shown, using the analytical results derived here, that all currently known LMBPs in globular clusters must have been affected by interactions, with their current eccentricities being at least an order of magnitude larger than at birth.

Distant interactions with passing stars may also be important for the orbital evolution of X-ray binaries in globular clusters. Hut \& Paczyński (1984) have shown that even a very small change in eccentricity can lead to a catastrophic increase in the mass transfer rate in a semidetached binary. In some cases, an eccentricity change as small as $\delta e \sim 10^{-4}-10^{-3}$ could even lead to the formation of a common envelope, ultimately destroying the binary. Other problems where our results could find applications include the interactions of binaries with massive black holes (Hills 1991) and the orbital evolution of a massive black-hole binary in a galactic nucleus (Mikkola \& Valtonen 1992). These problems involve extreme mass ratios $\left(m_{3} \gg M_{12}\right.$ or $\left.m_{3} \ll M_{12}\right)$ which we did not address specifically in this paper, but our analytical expressions should apply in those cases as well (cf. Fig. 9).

\section{ACKNOWLEDGEMENTS}

We are much indebted to Steve McMillan, whose numerical work put our investigations on the right track. We thank Sterl Phinney for useful comments on the manuscript and Piet Hut and Scott Tremaine for helpful discussions. This work was supported in part by NASA Grant HF-1037.01-92A while F.A.R. was a Hubble Fellow at the Institute for Advanced Study, Princeton. D.C.H. thanks the Institute for Advanced Study for its hospitality while work on this project was being carried out.

\section{REFERENCES}

Born M., 1960, The Mechanics of the Atom. Ungar, New York 
Burdet C.A., 1967, ZAMP, 18, 434

Byatt-Smith J.G.B., Davie A.M., 1990, Proc. R. Soc. Edin., 114A, 243

Camilo F., Nice D.J., Taylor J.H., 1993, ApJ, 412, L37

Danby J.M.A., 1962, Fundamentals of Celestial Mechanics. Macmillan, New York

Goldstein H., 1980, Classical Mechanics. Addison-Wesley, London

Heggie D.C., 1975, MNRAS, 173, 729

Hills J.G., 1975, AJ, 80, 809

Hills J.G., 1991, AJ, 102, 704

Hills J.G., Fullerton L.W., 1980, AJ, 85, 1281

Hut P., 1983, ApJ, 272, L29

Hut P., Paczyński B., 1984, ApJ, 284, 675

Hut P., McMillan S., Goodman J., Mateo M., Phinney E.S., Pryor C., Richer H.B., Verbunt F., Weinberg M., 1992, PASP, 104, 981

Lichtenberg A.J., Lieberman M.A., 1983, Regular and Stochastic Motion. Springer-Verlag, Berlin

McMillan S.L.W., 1996, in The Origins, Evolutions, and Destinies of Binary Stars in Clusters, ASP Conference Series, Vol. 90, eds. E.F. Milone, J.-C. Mermilliod, p. 413

Mikkola S., Valtonen M.J., 1992, MNRAS, 259, 115

Phinney E.S., 1992, Phil. Trans. R. Soc. Lond. A, 341, 39

Phinney E.S., Kulkarni S.R., 1994, ARAA, 32, 591

Plummer H.C., 1918, An Introductory Treatise on Dynamical Astronomy. CUP, Cambridge

Pollard H., 1976, Celestial Mechanics. Math. Assoc. Am.

Press W.H., Teukolsky S.A., Vetterling W.T., Flannery B.P., 1992, Numerical Recipes, Second Edition. CUP, Cambridge

Rappaport S., Putney A., Verbunt F., 1989, ApJ, 345, 210

Rasio F.A., Heggie D.C., 1995, ApJ, 445, L133

Spitzer L., Jr., 1987, Dynamical Evolution of Globular Clusters. Princeton UP, Princeton

Verbunt F., 1993, ARAA, 31, 93

\section{APPENDIX A: DERIVATION OF THE ANALYTICAL RESULTS}

\section{A1 Nonzero Initial Eccentricity}

Our task here is to derive equation (7) by integration of equation (6), where $\mathbf{F}$ is given by equation (4). At lowest order, which is all we shall need, we substitute unperturbed expressions for $\mathbf{r}$ and $\mathbf{R}$ on the right side of equation (4).

We denote by $\hat{\mathbf{a}}$ and $\hat{\mathbf{b}}$ two orthogonal unit vectors in the original plane of motion of the binary, such that $\hat{\mathbf{a}}$ is in the direction of pericentre. (In fact $\mathbf{e}=e \hat{\mathbf{a}}$.) We also let $\hat{\mathbf{c}}=\hat{\mathbf{a}} \times \hat{\mathbf{b}}$ be the unit vector in the direction of the angular momentum. Similarly we define two vectors $\hat{\mathbf{A}}$ and $\hat{\mathbf{B}}$ in the original plane of motion of $m_{3}$ relative to the barycentre of the binary.

Next, define coordinates $x, y, X$ and $Y$ such that $\mathbf{r}=x \hat{\mathbf{a}}+y \hat{\mathbf{b}}$ and $\mathbf{R}=X \hat{\mathbf{A}}+Y \hat{\mathbf{B}}$. Then expressions for these four coordinates in unperturbed Keplerian motion can be found in standard texts (e.g., Plummer 1918; Danby 1962). Thus $x=a(\cos E-e)$ and $y=b \sin E$ where $b=a \sqrt{1-e^{2}}$ and $E$ is the eccentric anomaly. It is related to time by Kepler's equation, $n\left(t-t_{0}\right)=E-e \sin E$, where

$n^{2} a^{3}=G M_{12}$,

and $t_{0}$ is the time of pericentre passage. If the relative motion of the third body is hyperbolic with eccentricity $e^{\prime}$, for this section and the next one the expressions are most conveniently written in polar form, i.e., $X=R \cos \theta$ and $Y=R \sin \theta$. Here the polar angle is related to time implicitly via angular momentum conservation, i.e. $R^{2} \dot{\theta}=\left[G M_{123} r_{\mathrm{p}}\left(e^{\prime}+1\right)\right]^{1 / 2}$, where $r_{\mathrm{p}}$ is the distance of closest approach of the third body to the barycentre of the binary. We take $t=0$ at this instant, i.e., when $\theta=0$. Also $R=r_{\mathrm{p}}\left(e^{\prime}+1\right) /\left(e^{\prime} \cos \theta+1\right)$.

The integration of the equation for $\dot{\mathbf{e}}$ proceeds by first averaging over the motion of the binary (at fixed $\mathbf{R}$ ). At this point, if only the quadrupole term $(n=2)$ is retained in equation (4), the result is

$\langle\dot{\mathbf{e}}\rangle=-\frac{\pi m_{3}}{M_{12} R^{5}} \frac{a^{2} b e}{T}\left[15 \xi \eta \hat{\mathbf{a}}+\left(-9 \xi^{2}+6 \eta^{2}+3 \zeta^{2}\right) \hat{\mathbf{b}}+3 \eta \zeta \hat{\mathbf{c}}\right]$

where $T$ is the period of the binary, and $\xi, \eta$ and $\zeta$ are defined implicitly by $\mathbf{R}=\xi \hat{\mathbf{a}}+\eta \hat{\mathbf{b}}+\zeta \hat{\mathbf{c}}$. In fact only the component of equation (6) along $\hat{\mathbf{a}}$ is needed, since $e \delta e=\mathbf{e} \cdot \delta \mathbf{e}=e \hat{\mathbf{a}} \cdot \delta \mathbf{e}$; and since $Y$ is an odd function of $t$ we find that the change in eccentricity is

$\delta e=-\frac{15 \pi m_{3}}{M_{12}} \frac{a^{2} b e}{T}\left(\hat{\mathbf{a}} \cdot \hat{\mathbf{A}} \hat{\mathbf{b}} \cdot \hat{\mathbf{A}} \int_{-\infty}^{\infty} \frac{X^{2}}{R^{5}} d t+\hat{\mathbf{a}} \cdot \hat{\mathbf{B}} \hat{\mathbf{b}} \cdot \hat{\mathbf{B}} \int_{-\infty}^{\infty} \frac{Y^{2}}{R^{5}} d t\right)$.

For hyperbolic motion of the third body the result is

$$
\begin{aligned}
\delta e= & -\frac{5 e}{2 e^{\prime 2}} \sqrt{\frac{m_{3}^{2} a^{3}\left(1-e^{2}\right)}{M_{12} M_{123} r_{\mathrm{p}}^{3}\left(1+e^{\prime}\right)^{3}}} \times \\
& \times\left\{\hat{\mathbf{a}} \cdot \hat{\mathbf{A}} \hat{\mathbf{b}} \cdot \hat{\mathbf{A}}\left[3 e^{\prime^{2}} \arccos \left(-\frac{1}{e^{\prime}}\right)+\left(4 e^{\prime 2}-1\right) \sqrt{{e^{\prime 2}}^{2}-1}\right]+\hat{\mathbf{a}} \cdot \hat{\mathbf{B}} \hat{\mathbf{b}} \cdot \hat{\mathbf{B}}\left[3{e^{\prime 2}}^{2} \arccos \left(-\frac{1}{e^{\prime}}\right)+\left(2{e^{\prime}}^{2}+1\right) \sqrt{{e^{\prime 2}}^{2}-1}\right]\right\}
\end{aligned}
$$


For parabolic motion $\left(e^{\prime} \rightarrow 1\right)$ the corresponding result is

$\delta e=-\frac{15 \pi}{8}\left(\frac{2 m_{3}^{2} a^{3}}{M_{123} M_{12} r_{\mathrm{p}}^{3}}\right)^{1 / 2} e \sqrt{1-e^{2}}(\hat{\mathbf{a}} \cdot \hat{\mathbf{A}} \hat{\mathbf{b}} \cdot \hat{\mathbf{A}}+\hat{\mathbf{a}} \cdot \hat{\mathbf{B}} \hat{\mathbf{b}} \cdot \hat{\mathbf{B}})$,

a result already given in Heggie (1975, eq. [5.66]), though with the wrong sign. It is convenient to write the direction cosines $\hat{\mathbf{a}} \cdot \hat{\mathbf{A}}, \hat{\mathbf{b}} \cdot \hat{\mathbf{A}}$, etc., in terms of the Keplerian elements outlined in the text. This leads to equations (7) and (8).

\section{A2 Initially Circular Binaries: The Power-Law Regime}

Both of these results vanish for an initially circular orbit. However, the octupole $(n=3)$ terms in equation (4) yield a nontrivial result in this case, which is the case we consider henceforth. As before we take $t=0$ at the instant of closest approach of $m_{3}$, but now we can choose $\hat{\mathbf{a}}$ to be in the direction of $\mathbf{r}$ at that time. The analogue of equation (A2) is

$\langle\dot{\mathbf{e}}\rangle=\frac{15 m_{3}\left(m_{1}-m_{2}\right) \pi a^{4}}{8 M_{12}^{2} T R^{5}}\left(1-5 \frac{\zeta^{2}}{R^{2}}\right)(\eta \hat{\mathbf{a}}-\xi \hat{\mathbf{b}})$

Converting to coordinates in the plane of motion of the third body, we find that

$\delta \mathbf{e}=\frac{15 m_{3}\left(m_{1}-m_{2}\right) \pi a^{4}}{8 M_{12}^{2} T}\left(\int_{-\infty}^{\infty} \frac{X^{3}}{R^{7}} d t\left[1-5(\hat{\mathbf{A}} \cdot \hat{\mathbf{c}})^{2}\right] \hat{\mathbf{A}} \times \hat{\mathbf{c}}+\int_{-\infty}^{\infty} \frac{X Y^{2}}{R^{7}} d t\left\{\left[1-5(\hat{\mathbf{B}} \cdot \hat{\mathbf{c}})^{2}\right] \hat{\mathbf{A}} \times \hat{\mathbf{c}}-10 \hat{\mathbf{B}} \cdot \hat{\mathbf{c}} \hat{\mathbf{A}} \cdot \hat{\mathbf{c}} \hat{\mathbf{B}} \times \hat{\mathbf{c}}\right\}\right)$.

Note that $\delta \mathbf{e}$ lies in the plane of motion of the binary, and is independent of the choice of $\hat{\mathbf{a}}$ and $\hat{\mathbf{b}}$ in this plane. Incidentally, it is also easily seen that $\delta \mathbf{e}$ is orthogonal to $\hat{\mathbf{A}}$ in certain circumstances, e.g., in coplanar motion. This means that the binary orbit becomes elongated in a direction orthogonal to the position of $m_{3}$ at pericentre.

For hyperbolic relative motion of the third body the two integrals in equation (A6) have the values

$\int_{-\infty}^{\infty} \frac{X^{3}}{R^{7}} d t=\frac{\sqrt{e^{\prime 2}-1}\left(2+11 e^{\prime^{2}}+32 e^{\prime^{4}}\right)+45 e^{\prime^{4}} \arccos \left(-1 / e^{\prime}\right)}{30 e^{\prime 3} \sqrt{G M_{123} r_{\mathrm{p}}^{5}\left(e^{\prime}+1\right)^{5}}}$

and

$\int_{\infty}^{\infty} \frac{X Y^{2}}{R^{7}} d t=\frac{\sqrt{{e^{\prime 2}}^{2}-1}\left(-2+9{e^{\prime 2}}^{2}+8 e^{\prime^{4}}\right)+15 e^{\prime^{4}} \arccos \left(-1 / e^{\prime}\right)}{30{e^{\prime}}^{3} \sqrt{G M_{123} r_{\mathrm{p}}^{5}\left(e^{\prime}+1\right)^{5}}}$.

From these results the values of $\delta \mathbf{e}$ and $\delta e=|\delta \mathbf{e}|$ are easily derived, though the expressions are relatively cumbersome. It helps to resolve $\delta \mathbf{e}$ along and perpendicular to the line of nodes, and to express the various products of unit vectors in terms of the elements $i$ and $\omega$ already introduced in $\S 2.2$. Eventually we obtain equation (9), where $f_{1}\left(e^{\prime}\right)$ is defined in equation (10). Note that, in the case of parabolic motion of the third body, we have $f_{1}(1)=\pi$, and so the result is rather more graceful.

\section{A3 Initially Circular Binaries: The Exponential Regime}

As we have seen, averaging over the fast motion of the binary leads to the conclusion that the quadrupole term gives a null result in the case of an initially circular binary, and that the octupole term provides the leading term in the induced eccentricity. At a sufficiently small distance of closest approach, however, the method of averaging becomes inaccurate, and so a different approach to the evaluation of the quadrupole term is needed. We continue, however, to restrict attention to an initially circular orbit.

In this case the contribution from the quadrupole term is

$\dot{\mathbf{e}}=\frac{m_{3}}{M_{12} R^{3}}\left[6 \frac{(\mathbf{r} \cdot \mathbf{R})(\dot{\mathbf{r}} \cdot \mathbf{R})}{R^{2}} \mathbf{r}-3 \frac{(\mathbf{r} \cdot \mathbf{R})^{2}}{R^{2}} \dot{\mathbf{r}}+r^{2} \dot{\mathbf{r}}\right]$.

As before we take $t=0$ at the time of closest approach of $m_{3}$, and choose the vector $\hat{\mathbf{a}}$ to be the direction of $\mathbf{r}$ at this instant, so that $\mathbf{r}=a(\hat{\mathbf{a}} \cos n t+\hat{\mathbf{b}} \sin n t)$, where $n$ is the mean motion of the binary.

Since the right side of equation (A7) involves the third power of components of $\mathbf{r}$ or $\dot{\mathbf{r}}$, its terms involve factors of exp $( \pm i n t)$ and $\exp ( \pm 3 i n t)$ when written in exponential form. From what follows we shall see that the resulting expressions for $\delta e$ would decrease like $\exp (-A)$ and $\exp (-3 A)$, respectively, where $A$ is proportional to $\left(r_{\mathrm{p}} / a\right)^{3 / 2} \gg 1$. Therefore we shall ignore at the outset the terms in $\exp ( \pm 3 i n t)$, which simply means that the method of averaging applies to them with greater accuracy because of their faster frequency. At this stage, then, we have

$\dot{\mathbf{e}}=\frac{m_{3} a^{3} n}{M_{12} R^{5}} \Re\left[e^{i n t} \hat{\mathbf{a}}\left(i R^{2}+\frac{3}{4} i \xi^{2}+\frac{9}{2} \xi \eta-\frac{15}{4} i \eta^{2}\right)+e^{i n t} \hat{\mathbf{b}}\left(R^{2}-\frac{15}{4} \xi^{2}+\frac{9}{2} i \xi \eta+\frac{3}{4} \eta^{2}\right)\right] ;$

here, as before, we have written $\mathbf{R}=\xi \hat{\mathbf{a}}+\eta \hat{\mathbf{b}}+\zeta \hat{\mathbf{c}}$, where $\hat{\mathbf{c}}=\hat{\mathbf{a}} \times \hat{\mathbf{b}}$. 
When we integrate with respect to $t$ we require integrals of the form

$I_{1}=\int_{-\infty}^{\infty} \frac{X_{i} X_{j}}{R^{5}} \exp ($ int $) d t$

where $X_{i}$ and $X_{j}$ are two of the non-zero components of $\mathbf{R}=X \hat{\mathbf{A}}+Y \hat{\mathbf{B}}$. Using two standard equations for hyperbolic motion, viz.

$n^{\prime} t=e^{\prime} \sinh F-F$,

and

$R=a^{\prime}\left(e^{\prime} \cosh F-1\right)$

where

$n^{\prime 2} a^{\prime^{3}}=G M_{123}$

we write this as

$I_{1}=\frac{1}{n^{\prime} a^{\prime}} \int_{-\infty}^{\infty} \frac{X_{i} X_{j}}{R^{4}} \exp \left[i \frac{n}{n^{\prime}}\left(e^{\prime} \sinh F-F\right)\right] d F$.

Now we recall the assumption that the encounter is slow (eq. [2]), which implies that the exponent here is rapidly oscillating compared with the time scale of variations of the other factors in the integrand. The asymptotic treatment of such integrals is standard. The idea is to evaluate the integral approximately by deforming the contour of integration off the real axis to the saddle point of the exponent ("method of steepest descents"), which occurs in this case at $R=0$, by equation (A11). (The process is rather like that adopted by Heggie 1975 in evaluating the change in energy, and the relevant contour is illustrated there.) The rest of the integrand in equation (A13) is singular here, however, and so we first reduce the singularity using partial integration. From equation (A11) we have $\frac{d R}{d F}=a^{\prime} e^{\prime} \sinh F$, and so equation (A13) becomes

$I_{1}=\frac{1}{3 n^{\prime} a^{\prime 2} e^{\prime}} \int_{-\infty}^{\infty} \frac{1}{R^{3}} \frac{d}{d F}\left\{\frac{X_{i} X_{j}}{\sinh F} \exp \left[i \frac{n}{n^{\prime}}\left(e^{\prime} \sinh F-F\right)\right]\right\} d F$

though we have already had to deform the contour to avoid the singularity at $F=0$. In performing the differentiation, to leading order we need only include the derivative of the exponential, since the factor $n / n^{\prime}$ brings down a factor of order $\left(r_{\mathrm{p}} / a\right)^{3 / 2}$. To leading order we therefore have

$I_{1}=\frac{i n}{3 G M_{123} e^{\prime}} \int_{-\infty}^{\infty} \frac{X_{i} X_{j}}{R^{2} \sinh F} \exp \left[i \frac{n}{n^{\prime}}\left(e^{\prime} \sinh F-F\right)\right] d F$,

where we have made use of equations (A11) and (A12).

A second application of the same trick leads to

$I_{1}=-\frac{n^{2}}{3 G M_{123}{a^{\prime 2}}^{\prime}{e^{\prime}}^{2}} \int_{-\infty}^{\infty} \frac{X_{i} X_{j}}{\sinh ^{2} F} \exp \left[i \frac{n}{n^{\prime}}\left(e^{\prime} \sinh F-F\right)\right] d F$,

and now we turn attention to the saddle point at $R=0$, i.e., $F=i \arccos \left(1 / e^{\prime}\right)$. In its vicinity we have

$e^{\prime} \sinh F-F \simeq i \sqrt{e^{\prime^{2}}-1}-i \arccos \frac{1}{e^{\prime}}+\frac{i}{2}\left(F-i \arccos \frac{1}{e^{\prime}}\right)^{2} \sqrt{e^{\prime 2}-1}$.

Now the integration is trivial, since in the rest of the integrand we can substitute values at the saddle, and so

$I_{1}=\frac{\sqrt{2 \pi}}{3} \frac{M_{12}^{3 / 4}}{G^{1 / 2} M_{123}^{5 / 4}} \frac{X_{i} X_{j}}{a^{9 / 4} r_{\mathrm{p}}^{5 / 4}\left(e^{\prime}+1\right)^{5 / 4}} \exp \left[-\left(\frac{M_{12}}{M_{123}}\right)^{1 / 2}\left(\frac{r_{\mathrm{p}}}{a}\right)^{3 / 2} \frac{\sqrt{e^{\prime 2}-1}-\arccos \left(1 / e^{\prime}\right)}{\left(e^{\prime}-1\right)^{3 / 2}}\right]$,

though we have yet to substitute values for $X_{i}$ and $X_{j}$ at the saddle point, where

$\mathbf{R}=\frac{r_{\mathrm{p}}\left(e^{\prime}+1\right)}{e^{\prime}}(\hat{\mathbf{A}}+i \hat{\mathbf{B}})$.

Now we return to equation (A8). Using equations (A18) and (A19) we readily find that

$$
\begin{aligned}
\mathbf{e}= & \frac{\sqrt{2 \pi} m_{3} M_{12}^{1 / 4} r_{\mathrm{p}}^{3 / 4}\left(e^{\prime}+1\right)^{3 / 4}}{3 M_{123}^{5 / 4} a^{3 / 4} e^{\prime 2}} \times \exp \left[-\left(\frac{M_{12}}{M_{123}}\right)^{1 / 2}\left(\frac{r_{\mathrm{p}}}{a}\right)^{3 / 2} \frac{\sqrt{e^{\prime 2}-1}-\arccos \left(1 / e^{\prime}\right)}{\left(e^{\prime}-1\right)^{3 / 2}}\right] \times \\
& \times\left\{\hat{\mathbf{a}}\left[-\frac{3}{2} \hat{\mathbf{a}} \cdot \hat{\mathbf{A}} \hat{\mathbf{a}} \cdot \hat{\mathbf{B}}+\frac{9}{2}(\hat{\mathbf{a}} \cdot \hat{\mathbf{A}} \hat{\mathbf{b}} \cdot \hat{\mathbf{A}}-\hat{\mathbf{a}} \cdot \hat{\mathbf{B}} \hat{\mathbf{b}} \cdot \hat{\mathbf{B}})+\frac{15}{2} \hat{\mathbf{b}} \cdot \hat{\mathbf{A}} \hat{\mathbf{b}} \cdot \hat{\mathbf{B}}\right]+\right. \\
& \left.+\hat{\mathbf{b}}\left[-\frac{15}{4}(\hat{\mathbf{a}} \cdot \hat{\mathbf{A}})^{2}+\frac{15}{4}(\hat{\mathbf{a}} \cdot \hat{\mathbf{B}})^{2}-\frac{9}{2}(\hat{\mathbf{a}} \cdot \hat{\mathbf{A}} \hat{\mathbf{b}} \cdot \hat{\mathbf{B}}+\hat{\mathbf{a}} \cdot \hat{\mathbf{B}} \hat{\mathbf{b}} \cdot \hat{\mathbf{A}})+\frac{3}{4}(\hat{\mathbf{b}} \cdot \hat{\mathbf{A}})^{2}-\frac{3}{4}(\hat{\mathbf{b}} \cdot \hat{\mathbf{B}})^{2}\right]\right\} .
\end{aligned}
$$


The scalar products here are easily evaluated in terms of the usual Keplerian elements $\omega, \Omega$ and $i$, where $\Omega$ is measured along the plane of motion of the binary from the direction of â to the ascending node, and $\omega$ is measured from the node to the direction of $\hat{\mathbf{A}}$. The last (geometric) factor in expression (A20) reduces, after some lengthy expressions, to the surprisingly compact expression

$\hat{\mathbf{a}}\left[-\frac{3}{2} \sin 2 \omega \sin ^{2} i+\frac{9}{4} \sin 2(\Omega+\omega)(1+\cos i)^{2}\right]+\hat{\mathbf{b}}\left[-\frac{3}{2} \cos 2 \omega \sin ^{2} i-\frac{9}{4} \cos 2(\Omega+\omega)(1+\cos i)^{2}\right]$,

whence the magnitude of the vector $\mathbf{e}$ is given by equation (12). When $e^{\prime}=1$ (parabolic motion) the exponential factor is $-\frac{2}{3}\left(\frac{2 M_{12}}{M_{123}}\right)^{1 / 2}\left(\frac{r_{\mathrm{p}}}{a}\right)^{3 / 2}$, and so we have arrived at equation (13).

\section{A4 Initially Circular Binaries: The Impulsive Limit}

We consider briefly the case in which the inequality in equation (2) is reversed. The duration of the encounter is then very short compared with the binary period. This case appears unimportant for most astrophysical applications, and we include it here mainly for the discussion in Section 3.2.

In equation (6) we model $\mathbf{F}$ as a delta function, and then this equation integrates to

$G M_{12} \mathbf{e}=2(\delta \dot{\mathbf{r}} \cdot \dot{\mathbf{r}}) \mathbf{r}-(\delta \dot{\mathbf{r}} \cdot \mathbf{r}) \dot{\mathbf{r}}$,

where $\delta \dot{\mathbf{r}}$ is the change in relative velocity of the components due to the encounter. (The second term in eq. [6] vanishes for a circular binary.) We evaluate $\delta \dot{\mathbf{r}}$ by integrating the quadrupole term of equation (4), keeping $\mathbf{r}$ fixed and taking for $\mathbf{R}$ the form corresponding to a rectilinear orbit. In the notation of Appendix A1 this is $\mathbf{R}=r_{\mathrm{p}} \hat{\mathbf{A}}+V t \hat{\mathbf{B}}$, if $t=0$ at the time of pericentre. It follows that

$\delta \dot{\mathbf{r}}_{i}=\frac{G m_{3} r_{j}}{V r_{\mathrm{p}}^{2}}\left(4 \hat{A}_{i} \hat{A}_{j}+2 \hat{B}_{i} \hat{B}_{j}-2 \delta_{i j}\right)$,

where summation over $j$ is implied, and the $\delta$ on the right is the Kronecker symbol.

When this result is substituted into equation (A22) we also write $\mathbf{r}=a \hat{\mathbf{a}}$ and $\dot{\mathbf{r}}=n a \hat{\mathbf{b}}$, where the unit vectors have the meaning assigned to them in $\S \mathrm{A} 2$, and $n$ is defined in equation (A1). It quickly follows that

$G M_{12} \mathbf{e}=\frac{G m_{3} n a^{3}}{V r_{\mathrm{p}}^{2}}\left\{4(2 \hat{\mathbf{a}} \cdot \hat{\mathbf{A}} \hat{\mathbf{b}} \cdot \hat{\mathbf{A}}+\hat{\mathbf{a}} \cdot \hat{\mathbf{B}} \hat{\mathbf{b}} \cdot \hat{\mathbf{B}}) \hat{\mathbf{a}}-2\left[2(\hat{\mathbf{a}} \cdot \hat{\mathbf{A}})^{2}+(\hat{\mathbf{a}} \cdot \hat{\mathbf{B}})^{2}-1\right] \hat{\mathbf{b}}\right\}$

The two terms are orthogonal, and so $e$ is easily found.

In the case of coplanar motion, we introduce the angle $\theta$, defined to be the angle between the vectors $\hat{\mathbf{a}}$ and $\hat{\mathbf{A}}$. Then the direction cosines are easily expressed in terms of $\theta$, and equation (28) follows easily.

\section{APPENDIX B: THE EQUAL-MASS CASE}

The purpose of this appendix is to show that, for a circular binary with components of equal mass $m$, the induced eccentricity vanishes to all orders of the perturbation parameter $a / r_{\mathrm{p}}$. In first order perturbation theory this is easy to understand. All terms in equation (4) with $n$ odd contribute nothing if $m_{1}=m_{2}$. Hence $\mathbf{F}$ contains only odd monomials in the components of the vector $\mathbf{r}$, and then it is easy to see that the right side of equation (6) vanishes after averaging over one binary period, if $e=0$. The following argument extends this reasoning to perturbation theory at all orders.

Higher-order perturbation theory is best addressed using Hamiltonian methods, and here we describe the appropriate variables in the simplest non-trivial case: coplanar motion of all three stars. The relative motion of the binary components is conveniently handled in action-angle variables, which, for planar Keplerian motion, can be taken as equivalent to the classical Delaunay variables $L, G, l$ and $g$ (cf. Plummer 1918). The variables $L$ and $G$ are expressible in terms of the semi-major axis $a$ and eccentricity $e$ by means of

$a=2 L^{2} /\left(G m^{3}\right)$

and

$G=L \sqrt{1-e^{2}}$.

The conjugate variable $l$ is the mean anomaly, which increases uniformly with time in unperturbed motion, passes the value 0 at pericentre, and increases by $2 \pi$ in each revolution. The variable $g$ is the longitude of the pericentre, which we assume is measured from the direction of one axis of an inertial frame. The Hamiltonian function for unperturbed Keplerian motion is a function of $L$ alone, denoted by $H_{b}(L)$.

The motion of the third star we continue to describe by its position vector $\mathbf{R}$ with respect to the barycentre of the binary, and we introduce the conjugate momentum $\mathbf{P}$. Then the Keplerian approximation to its motion can be described by 
a Hamiltonian function $H_{3}(\mathbf{R}, \mathbf{P})$.

The whole Hamiltonian can now be written as

$H=H_{b}(L)+H_{3}(\mathbf{R}, \mathbf{P})+\Re(\mathbf{r}(L, G, l, g), \mathbf{R})$,

where the three terms on the right correspond, respectively, to the binary, the Keplerian motion of the third body, and the perturbation. The last of these terms is, except for the terms with $n=0$ and 1 and a factor involving the masses, nothing other than the expression whose gradient appears in equation (4), but we write $\mathbf{r}$ in terms of the Delaunay variables.

In the case of equal masses, $\Re$ is invariant under the transformation $\mathbf{r} \rightarrow-\mathbf{r}$, i.e. the transformation $g \rightarrow g+\pi$, with the other three canonical variables unchanged. To see that these transformations are equivalent, it is useful to consider the case of a binary of low eccentricity. Approximate expressions for the relative position vector $\mathbf{r}$ of the two components are easily found (cf. Plummer 1918 again) as

$\mathbf{r}=a\left(\cos l-\frac{3}{2} e+\frac{1}{2} e \cos 2 l, \sin l+\frac{1}{2} e \sin 2 l\right)$,

if the components are resolved along the directions of the major and minor axes of the relative orbit, and

$\mathbf{r}=a\left(\cos (g+l)-\frac{3}{2} e \cos g+\frac{1}{2} e \cos (2 l+g), \sin (g+l)-\frac{3}{2} e \sin g+\frac{1}{2} e \sin (2 l+g)\right)$,

relative to the same frame as that in which $g$ is measured. The effect of the transformation $g \rightarrow g+\pi$ is obvious.

Because $g$ is ill-defined when $e$ becomes very small, it is better to use the Poincaré variables $L^{\prime}, l^{\prime}, \xi$ and $\eta$, defined by $L^{\prime}=L, l^{\prime}=l+g$,

$\xi=\sqrt{2 G^{\prime}} \cos g$

and

$\eta=-\sqrt{2 G^{\prime}} \sin g$

where

$G^{\prime}=L-G \simeq(1 / 2) e^{2} L$,

by equation (B2). These are still canonical, $l^{\prime}$ and $\eta$ being conjugate to $L^{\prime}$ and $\xi$, respectively. In these variables equation (B5) becomes

$\mathbf{r}=a\left(\cos l^{\prime}-\frac{3}{2} \frac{\xi}{\sqrt{L^{\prime}}}+\frac{1}{2 \sqrt{L^{\prime}}}\left(\xi \cos 2 l^{\prime}-\eta \sin 2 l^{\prime}\right), \sin l^{\prime}+\frac{3}{2} \frac{\eta}{\sqrt{L^{\prime}}}+\frac{1}{2 \sqrt{L^{\prime}}}\left(\xi \sin 2 l^{\prime}+\eta \cos 2 l^{\prime}\right)\right)$

approximately, and the transformation $l^{\prime} \rightarrow l^{\prime}+\pi, \xi \rightarrow-\xi, \eta \rightarrow-\eta$ sends $\mathbf{r} \rightarrow-\mathbf{r}$.

In general, $\mathbf{r}$ is a $2 \pi$-periodic function of $l^{\prime}$, and can be expanded as a Fourier series $\mathbf{r}=\sum_{n} \mathbf{r}_{n}\left(L^{\prime}, \xi, \eta\right) \exp \left(i n l^{\prime}\right)$, where the coefficients $\mathbf{r}_{n}$ are actually power series in $\xi$ and $\eta$. This is illustrated at lowest non-trivial order by equation (B9). Similarly, we may expand the perturbing function as a series $\Re=\sum_{-\infty}^{\infty} a_{n}\left(L^{\prime}, \xi, \eta, \mathbf{R}\right) \exp \left(i n l^{\prime}\right)$, where again the coefficients are power series in the variables $\xi$ and $\eta$. Now the invariance of the Hamiltonian under the transformation $\mathbf{r} \rightarrow-\mathbf{r}$ (for equal masses) implies that this series is invariant under the transformation $l^{\prime} \rightarrow l^{\prime}+\pi, \xi \rightarrow-\xi, \eta \rightarrow-\eta$. If the coefficient $a_{n}$ contains a term $\xi^{\alpha} \eta^{\beta}$ it follows that $\alpha+\beta$ is even if $n$ is even, and $\alpha+\beta$ is odd if $n$ is odd.

The aim of Hamiltonian perturbation theory is to remove those terms which depend on quickly-varying angles. At lowest order, this is equivalent to averaging over the fast motions. Since $l$ or $l^{\prime}$ is the one rapidly changing quantity, this leaves only those terms in the Fourier series which have $n=0$. These terms have as coefficients power series in $\xi$ and $\eta$ which have even "order" (i.e. the value of $\alpha+\beta$ ). When these are differentiated with respect to these variables (to give Hamilton's equations for their evolution) the resulting expression has terms of only odd order in these variables. It follows, therefore, that one solution is $\xi=\eta=0$, and since this solution yields the correct initial condition for a circular binary, it follows that the induced eccentricity vanishes (in first order perturbation theory).

All we have to do now is to show that terms of the wrong parity will not be introduced in the perturbation procedure. In the standard procedure, which is described in many texts (e.g. Born 1960; Goldstein 1980; Lichtenberg \& Liebermann 1983), the terms with $n \neq 0$ are removed by a near-identity canonical transformation. For example, a term $a_{n} \exp \left(i n l^{\prime}\right)$ is removed by a transformation to new variables (denoted here by stars) in which

$l^{\prime \star}=l^{\prime}+\frac{\partial S_{1}}{\partial L^{\prime \star}} e^{i n l^{\prime}}, \quad \xi=\xi^{\star}+\frac{\partial S_{1}}{\partial \eta} e^{i n l^{\prime}}$,

etc., where

$S_{1}\left(\eta, \mathbf{R}, L^{\prime \star}, \xi^{\star}, \mathbf{P}^{\star}\right)=-\frac{a_{n}\left(\eta, \mathbf{R}, L^{\prime \star}, \xi^{\star}, \mathbf{P}^{\star}\right)}{i n \frac{\partial H_{b}\left(L^{\prime \star}\right)}{\partial L^{\prime \star}}}$ 
(cf. Lichtenberg \& Lieberman 1983, eq. [2.2.17]).

Now it is clear from inspection of these equations (and the corresponding equations for the transformation of the other variables), along with our result on the parity of the coefficient $a_{n}$, that the transformation $l^{\prime} \rightarrow l^{\prime}+\pi, \xi \rightarrow-\xi, \eta \rightarrow-\eta$ corresponds, in the new variables, to the transformation $l^{\prime \star} \rightarrow l^{\prime \star}+\pi, \xi^{\star} \rightarrow-\xi^{\star}, \eta^{\star} \rightarrow-\eta^{\star}$. Since the Hamiltonian was invariant under the transformation in the old variables, it will also be invariant under the transformation in the new ones. It follows that, when the Hamiltonian is expressed in terms of the new variables, the new coefficients $a_{n}$ will have the same parity property as the old ones. It follows that the induced eccentricity will again vanish for an initially circular binary. By induction, this conclusion follows for perturbation theory at all orders. 